\title{
The Firm as the Locus of Social Comparisons: Standard Promotion Practices versus Up-or-out*
}

\author{
Emmanuelle Auriol \\ Toulouse School of Economics \\ CEPR
}

\author{
Guido Friebel \\ Goethe University Frankfurt \\ CEPR, IZA
}

\author{
Frauke von Bieberstein \\ University of Bern
}

September 2015

\begin{abstract}
We suggest a parsimonious dynamic agency model in which workers have status concerns. A firm is a promotion hierarchy in which a worker's status depends on past performance. We investigate the optimality of two types of promotion hierarchies: (i) standard promotion practices, where agents have a job guarantee, and (ii) "up-orout", in which agents are fired when unsuccessful. We show that up-or-out is optimal if success is difficult to achieve. When success is less hard to achieve, standard promotion practices are optimal provided the payoffs associated with success are moderate. Otherwise, up-or-out is, again, optimal.
\end{abstract}

Keywords: Status, promotion hierarchies, incentives, sorting JEL Classification Numbers: J3, M5, L2

\footnotetext{
*Corresponding author: Guido Friebel, Goethe University Frankfurt, Graneburgplatz 160323 Frankfurt am Main. +496979834826; gfriebel@wiwi.uni-frankfurt.de. We would like to thank seminar and conference participants in Bonn, the Personnel Economics Conference in Trier, the Rotterdam TI / Mannheim ZEW workshop on Social Relations and Incentives in the Workplace, and the Bad Homburg Workshop on Organizational Economics. In particular we thank Robert Dur, Matthias Kräkel, Susanne Neckermann, and Ferdinand von Siemens for helpful suggestions. We gratefully acknowledge support from ANR and DFG, von Bieberstein also from the Volkswagen Foundation, Grant no. 85 487. Parts of the paper were written while von Bieberstein was visiting the Toulouse School of Economics. All errors are ours.
} 


\section{Introduction}

Status is an important determinant for human behavior, a proposition supported by psychologists and economists alike (e.g. Frank, 1988, Huberman et al., 2004, Moldovanu et al., 2007). Status concerns are particularly important at work; people spend much of their time at the workplace, and their behavior at work is an important determinant of an economy's efficiency. The goal of gaining higher status in an organization motivates people to work hard for long periods of time; examples from the academic world, law firms, investment banks, and consulting firms abound. Making partner at a law firm, or getting tenure in a university provides much stronger motivation than just getting a wage rise.

Chester Barnard (1938, p.145), the first modern management theorist, was well aware of the relevance of status for motivation and the necessity to provide both monetary rewards and status: "Even in strictly commercial organizations, where it is least supposed to be true, money without distinction, prestige, position, is so utterly ineffective that it is rare that greater income can be made to serve even temporarily as an inducement if accompanied by suppression of prestige." Peter Drucker (1954, p. 154) expressed similar thoughts: "But financial rewards are not enough. People, whether managers or workers, whether in business or outside, need rewards of prestige and pride." Indeed, most organizations do not only provide monetary incentives, but they also allocate status between workers by giving them awards, office space, company cars, and, arguably most importantly, promotions. Some of these attributes also provide material benefits or affect working conditions, whereas others are purely symbolic and are valued for the social or psychological benefits they entail. While we acknowledge all of these factors, for the sake of clarity and tractability, in this paper we focus on the non-material sources of status.

Despite the prominence of status concerns and the widespread use of promotions as a way to differentiate people's status, there are relatively few papers in economics investigating the design of organizations and incentive contracts in the presence of status concerns (Auriol and Renault 2001, 2008; Moldovanu et al, 2007; Besley and Ghatak, 2008; Marino and Ozbas, 2011). ${ }^{1}$

Our paper builds on Auriol and Renault (2001, 2008). Auriol and Renault (2008) propose a general representation of preferences over social status and income where both tend to reinforce each other. Given these preferences, they show that a principal who can commit will always offer minimum rewards (both in status and money) to junior employees. Juniors will be incentivized to work only through the perspective of promotion. Auriol and Renault (2001) take a particular example of this general framework to derive closed form solutions of the optimal incentive contract. In these papers, though, workers are homogeneous and do not get fired, even if they are unsuccessful. In reality, workers differ in their productivity and people may get fired. In the present paper, we allow for such firing policies as part of the optimal hierarchy, and compare what has been called "standard promotion practices"

\footnotetext{
${ }^{1}$ Status in Auriol and Renault $(2001,2008)$ and Besley and Ghatak (2008) is different from Moldovanu et al. (2007), in which money is the source of status. As we are interested in organizational design, it is natural to focus on rank in a hierarchy as the source for status.
} 
with the "up-or-out system".

Going back to Doeringer and Piore (1971), in firms employing standard promotion practices incentives are given through promotions and wages are associated to job titles (see for instance, Baker et al., 1994). "Up-or-out" exposes employees to more risk and steeper incentives. Employees work for some years as juniors with the explicit or implicit understanding that upon completion of this phase, they will either be promoted or will have to leave the firm.

We derive the two promotion hierarchies as solutions to a simple dynamic agency model, describe their properties in terms of profits, wages paid to juniors and seniors, and the ratio between them, the implied span of control. We determine when one or the other is optimal and generate some empirical predictions. We also consider heterogeneous workers and endogenize workers' outside options by considering self-employment opportunities.

An important literature has looked at the rationale for firms to maintain standard promotion practices, ${ }^{2}$ and up-or-out has attracted much attention among economists as well. ${ }^{3}$ As in Lazear and Rosen (1981) or in Malcomson (1984), much of the literature on promotion hierarchies argues that tournaments solve contracting issues related to non-verifiable or hardly measurable output.

Our model is also a tournament model, because successful workers receive increases in wages and status, but we do not rely on restrictions on the contractibility of output: output is verifiable and measurable on a cardinal scale. Indeed, in many sectors in which up-orout is applied, measures of individual output are observable and verifiable. For instance, in academia, publications and citations of a researcher are easily verifiable. Although these might be imperfect measures of productivity, they are employed as a proxy for individual output and are not only used as the basis for promotion decisions taken by universities, but also for the allocation of public funds (e.g. in Germany and the UK). Similar measures are available for the record of investment bankers (the return on the funds invested or the profits generated) or lawyers (size and frequency of cases won, or clients acquired).

In our model, there is one large firm that offers workers either standard promotion practices, or up-or-out. Workers can choose to be self-employed or to work in the large firm. Three different employment forms are hence generated from the same model: self-employment, standard promotion practices, and up-or-out systems. By working in the promotion hierarchy, workers receive the opportunity of gaining additional status. The firm allocates status among workers by means of job titles or ranks. For incentive purposes, all juniors enjoy the same low status and successful agents receive a large increase in status through promotions. In equilibrium, only high-productivity agents enter the large firm and as everyone exerts the same effort, workers take a status gamble. Self-employment, in which there is no reallocation of status, ${ }^{4}$ is preferred by less productive workers, because they have little to gain

\footnotetext{
${ }^{2}$ For surveys of the theoretical and empirical literature, see Gibbons and Waldman (1999), and Waldman (2009).

${ }^{3}$ Below we review the relationship to the following papers: Demougin and Siow (1994), O'Flaherty and Siow (1995), Kahn and Huberman (1988), Waldman (1990), Ghosh and Waldman (2010).

${ }^{4}$ Notice that we are not saying that there are no social status concerns of self-employed, as the market
} 
from competing for status and incentive pay with more productive types. Note that in our framework we do not consider highly visible entrepreneurs like Bill Gates and Mark Zuckerberg, who are the principals of large corporations. Instead, we have in mind self-employed entrepreneurs with low visibility working on their own and employing no one else. ${ }^{5}$ Thus, promotion hierarchies function as a screening device ensuring that only productive workers enter a firm, and firms make profits by using promotion hierarchies. ${ }^{6}$

The large firm makes profits because junior workers receive wages below the output they produce. They enjoy little status, but they face steep incentives by the prospect of receiving both a large fixed wage, a substantial bonus and high status in the firm when promoted upon a success. Thus, both junior and senior workers are exerting more effort than in self-employment. These productivity gains are shared between the firm and the successful workers who are promoted. An entrepreneur who would hire only one worker or hire more than one worker without differentiating their status would not make profits because workers have the alternative to be self-employed. In the absence of status differentiation, the optimal dynamic solution would simply be the replication of the optimal static solution. ${ }^{7}$

We derive the optimal incentive and promotion scheme both for standard promotion practices and for up-or-out systems. We identify the situations in which standard promotion practices and those in which up-or-out contracts are optimal. We find that in terms of the profits of the firm, up-or-out is always optimal if it is very difficult to achieve a success. When success is less hard to achieve, standard promotion practices are optimal provided the payoff associated with success is small enough. Otherwise up-or-out is, again, optimal.

The intuition of our results is the following: Large firms can exploit the complementarity between status and income in workers preferences by bundling high status and high income. This lowers the total wage bill. Wages and status are shifted to the second period of the professional life to provide the workers with incentives to work, explaining the backloaded

for Rolex or Porsche demonstrates. However, such external symbols are different from the internal status symbols like promotions. Internal status can be controlled by the firm and is, consequently, an element of organizational design, which is the focus of our paper.

${ }^{5}$ These small enterprises are not able to redistribute status inside the firm. Indeed, social status stems from interpersonal comparisons, in our case embodied in the formal ranking of workers inside the hierarchy that can be controlled by the principal. There is, however, no possibility to manage social status when one works alone (i.e., there is nobody to compare with). This is why, with self-employment, social status is fixed. The model hence compares competition between small and large firms all of which provide status internally. Small firms are limited in what they can do in terms of status allocation. By contrast, there is ample room for status manipulation in large firms by assigning ranks. The principal chooses the status allocation to maximize profit (i.e., she is assumed to care only for profit as shareholders would).

${ }^{6}$ This is a similar mechanism as the one in von Siemens (2010) in which a firm can prevent inequity-averse low-ability workers from entering by not compensating them for rent differences within the firm.

${ }^{7}$ We believe this result to be (technically) interesting because Ohlendorf and Schmitz (2012) show in the same setting that the optimal dynamic contract displays memory. We show that their result hinges on the fact that in their model, the workers' reservation utility is fixed to 0, implying that only the limited liability constraint is binding. In our model, however, the outside option of the workers is type-dependent (agents differ in productivity), so that the participation constraint binds in equilibrium. Given that agents are riskneutral and that in the outside option workers receive all of the surplus they produce, the firm cannot do better than this contract by postponing rewards. 
structure of the dynamic incentive scheme. While it would seem that up-or-out is always the preferable scheme because it offers steeper incentives to juniors, this is not the case.

In particular, when effort has little value, incentives can be too steep in up-our-out. The reason is that the firm can only adjust the status of people working inside the company. Thus, when up-or-out requires unsuccessful juniors to leave, this forces the status of these workers to the minimum that is earned in self-employment. In turn, the firm needs to give a higher status and income to successful seniors to fulfill the participation constraint of the junior workers, at the same time increasing incentives for juniors. Juniors might then spend too much effort which has to be compensated by the firm. Therefore, when incentives to juniors are less important and the main driver of profits is the intergenerational redistribution of status, standard promotion practices based on seniority are preferable.

Our theory generates a number of other predictions. The variance of career success (measured in wages and status) of a cohort entering a firm with standard promotion practices should be lower than the one of a cohort entering an up-or-out hierarchy. Juniors should work harder in up-or-out than with standard promotion practices; while this is not necessarily so for seniors. The model also predicts differences in the spans of control in up-or-out and with standard promotion practices: the ratio of juniors and (in the case of standard promotion practices) unsuccessful seniors over successful (promoted) seniors is smaller in up-or-out than with standard promotion practices.

In our model, both promotion hierarchies induce sorting with only productive workers entering the large firm. As we know from the empirical literature (for instance, Lazear, 2000), firms must be concerned about both, effort provision and sorting of productive workers. We find it noteworthy that status differentiation serves this double purpose in a similar way as incentive pay.

The next section relates our paper to the literature; Section 3 sets up the model; Section 4 presents the main results. Section 5 derives implications and discusses the results and Section 6 concludes.

\section{Related literature}

We share a common interest with the existing papers on up-or-out contracts, but there are some notable differences, both in terms of underlying assumptions and predictions. First, Demougin and Siow (1994) and O'Flaherty and Siow (1995) are not about incentives. Rather, firms decide on either to staff all junior positions with trainees for managerial positions only, or to staff the junior positions also with people who work productively. Whether or not upor-out is optimal depends on demand. In particular, if current demand is low, but growth is high, up-or-out is optimal. We consider a steady-state organization, and in our theory, juniors and seniors do the same kind of work, which seems a fair description of law firms, consulting or academia. The main interest that relates us to Demougin and Siow (1994) and O'Flaherty and Siow (1995) lies in determining the relative sizes of different hierarchical levels and, thus, the span of control of hierarchies. 
The second group of papers is on incentives. Kahn and Huberman (1988) suggest a model in which firms want to incentivize agents to invest in human capital, but there is limited commitment of the firms. Output is only observable to the firm; it thus may pretend that the output is not high enough in order to save on the promised reward. This would undermine the incentive effects of the proposed reward. By announcing that anyone who does not get the reward will be fired, the firm can commit itself not to cheat, because otherwise it will lose the accumulated human capital of the worker. The model combines bilateral moral hazard with the assumption that output cannot be verified. Prendergast (1993) suggests a model in which promotions, together with wage structures that a firm can commit itself to, can solve similar problems related to unverifiability and specific human capital acquisition. The assumption of unverifiable output is also present in Waldman (1984) and (1990) and in Ghosh and Waldman (2010) in which promotion is a signal to the outside world about the productivity of a person, while output is not observable to the outside world. Waldman (1984) introduced this idea and then showed (Waldman, 1990) that the Kahn and Huberman (1988) model works in a setting with general human capital if promotion as a signal is considered. Ghosh and Waldman (2010) compare standard promotion practices with up-or-out, and show that up-or-out is optimal if firm-specific human capital is low. They also show that if the firm can commit to a wage floor, up-or-out is used when low and high-level jobs are similar.

An ongoing question in the literature on up-or-out is thus the impact of firm-specific human capital on the optimal promotion system. While the model of Kahn and Huberman (1988) predicts that up-or-out should prevail when firm-specific human capital is important, the model of Ghosh and Waldman (2010) predicts the opposite. Gorman (1999) presents empirical evidence that is in line with the results in Ghosh and Waldman (2010). We will argue later that introducing firm-specific human capital in our model would produce similar results, i.e., up-or-out would become less attractive.

\section{3. $\quad$ Model}

We employ an overlapping generation model. At any date, the organization is staffed with members of two generations. Each person has a work life time of two periods. Juniors either enter the organization and work their first period or stay out. Seniors who joined the organization in the previous period may spend the last period of their working life in the firm, or work in self-employment. ${ }^{8}$ It is assumed that the population of agents is constant and large so that it may be represented by a continuum. The size of the workforce employed by the firm is normalized to 2 , that is, we look at a firm in steady state.

\footnotetext{
${ }^{8}$ In a similar setup without considering status concerns, Bar-Isaac (2007) examines juniors and seniors working together in teams to credibly establish a reputation for working hard.
} 
The risk-neutral organizational designer maximizes the profit over two periods:

$$
\sum_{t=1}^{2} \delta^{t} \pi_{t}=\sum_{t=1}^{2} \delta^{t}\left(Q_{t}-W_{t}\right) .
$$

where $Q_{t}$ is output (its price is normalized to 1 without loss of generality) and $W_{t}$ is the wage bill in period $t$. The principal's objective function is intertemporally separable with a discount factor $\delta \leq 1$. In what follows, we set $\delta=1$ which is innocuous in our context.

The organizational designer uses two instruments, compensation and allocation of status, both of which are perfectly observable. Before setting up the full program of the designer, we describe the production process, workers' preferences, and the feasible allocations of status.

\subsection{Production}

Each worker living at date $t$, junior or senior, exerts an effort $e_{i t} \geq 0$ through which he contributes an amount $q_{i t}$ to the firm's output. Workers are hired to do the same type of work. With probability $\mu\left(e_{i t}\right), q_{i t}$ is high $\left(q_{i t}=q+\Delta q, \Delta q>0\right)$, and with probability $1-\mu\left(e_{i t}\right), q_{i t}$ is low $\left(q_{i t}=q\right)$. Thus, $q$ is the base-line output, and the total output at date $t, Q_{t}=\int_{0}^{2} q_{i t} d i$, is random. Individual output $q_{i t}$ is verifiable and its realization is independent across time. The probability of high output of agent $i, \mu\left(e_{i t}\right)$, increases with $e_{i t}$. More specifically we assume that

$$
\mu\left(e_{i t}\right)=\min \left\{e_{i t}, 1\right\}, \forall e_{i t} \geq 0 .
$$

While output $q_{i t}$ is ex-post verifiable, the effort level $e_{i t}$ is not. There are two types of workers, $g$ and $b$. A worker's type is private information. Type $g$ workers are more productive than type $b$ workers. The disutility of effort is

$$
\psi_{\tau}\left(e_{i t}\right)=\frac{\left(e_{i t}\right)^{2}}{2} a_{\tau}, \tau=g, b .
$$

The larger is $a_{\tau}$, the more difficult it is for an agent to achieve a high output. ${ }^{9}$ In equilibrium the probability of success is inversely related to $a_{\tau}$. To capture the idea that $g$ workers are more productive than $b$ workers we assume that $a_{b}>a_{g}>0$.

\section{$3.2 \quad$ Preferences}

We assume that utilities are additively separable across periods with some discount factor (which we will set to one). Workers are protected by limited liability and status and wages are publicly observable. In any of the periods an agent with productivity of type $\tau$, with

\footnotetext{
${ }^{9}$ Equivalently, we could assume $\mu_{\tau}(e)=\min \left\{\frac{e}{a_{\tau}}, 1\right\}$ and $\psi_{\tau}(e)=\frac{e^{2}}{2}$, with similar results.
} 
status $s \geq 0$, total income $\underline{w} \geq 0$, and effort level $e \geq 0$ has the following utility function:

$$
U_{\tau}(s, \underline{w}, e)=s \underline{w}-\psi_{\tau}(e) .
$$

We posit the same utility function as Auriol and Renault (2008), which is situated between perfect substitutes and perfect complements. The indifference curves for money and status for a given effort level are strictly decreasing, thus there is some substitution between status and income. This substitution is however imperfect: a superb job title does not compensate for a wage of nil, nor does a stellar wage make up for a lack of appreciation by others. The utility function also implies that the marginal rate of substitution between effort and income is decreasing in status. Put differently, for a given level of monetary incentives, an agent should be all the more willing to exert effort when she has higher status. Furthermore, the marginal rate of substitution between effort and status is decreasing in income. Thus, individuals with higher income will be willing to exert more effort in order to improve their status. Maslow's (1954) hierarchy of needs is in line with this, and Centers and Bugental (1966) find evidence that employees earning higher wages care more for factors at the top of Maslow's hierarchy of needs.

These observations indicate that our assumption is a reasonable one, but direct empirical evidence on the shape of utility functions is hard to find. While some authors in the literature on status have conjectured that the complementarities we assume exist (for instance, Neckermann and Kosfeld, 2011), systematic evidence about the interplay between status and social recognition, and money is so far lacking in economics. ${ }^{10}$ Evidence on the re-enforcement of money and social recognition exists in industrial psychology. In particular, Stajkovic and Luthans (2003) carry out a meta-analysis of 72 studies with more than 13,000 subjects. They find that provided that there is performance feedback (as in our theory in which performance is common knowledge), monetary incentives and social recognition re-enforce each other, as postulated in our theory.

\subsection{Organizational design}

An organization can establish a status ranking of their workers through different means like the distribution of wages, the allocation of scarce nonmonetary resources, e.g., corner offices, or, most commonly, the hierarchical structure. Some of these attributes also provide material benefits, whereas others are purely symbolic and are valued for the social or psychological benefits they entail. We focus on these non-material sources of status, such as rank in the organization. In particular, we consider rank as a pure status device rather than a reflection of different responsibilities (as analyzed by Garicano, 2000) or span of control (as analyzed by Smeets et al, 2013, both of which are complementary issues to our analysis.

Any organization will be constrained in its allocation decision because increasing one

\footnotetext{
${ }^{10}$ A first piece of related evidence is by Bradler and Neckermann (2011) who found that in a field experiment a monetary reward and a thank-you card increased performance to some limited extent when given in combination. This, however, is only mild support as a promotion is not given to everyone.
} 
individual's status comes at the expense of decreasing somebody else's status. We thus assume that status is firm-specific and that each worker is born with one unit of status. The set of feasible social status allocations is characterized as follows (the equality to 2 is a normalization).

$$
\int_{0}^{2} s_{i t} d i=2, s_{i t} \geq 0 \forall i, t
$$

For each agent, the organizational designer chooses a social status allocation $s_{i t}$ in (A.1), a fixed wage $w_{i t}$, and a bonus $\Delta w_{i t}$ in case of a high performance. Status is allocated before the workers exert effort. When an agent joins the organization he is assigned to a rank in the hierarchy, which is revised at the end of the first period based on performance.

The assumption that the firm is free to assign status in any possible way is certainly a strong one. However, a number of papers in psychology (Jemmott and Gonzalez, 1989) and economics (Ball and Eckel, 1998; Ball et al., 2001) have shown that even perfectly randomly distributed status affects people's performance. For instance, Ball et al. (2001) awarded a "gold star" (a pin) to half of the participants in a standard buyer/seller game. Status was found to have a significantly positive effect on individuals' earnings, even though participants knew that status was awarded on an arbitrary basis.

We will focus on the two promotion hierarchies most commonly observed in practice. First, in an up-or-out system, successful former juniors are promoted and become seniors, while unsuccessful juniors have to leave. Second, with standard promotion practices, again, successful juniors become seniors, but unsuccessful juniors are offered to stay in the firm and become seniors. We will show though that these "unsuccessful" seniors (who were unsuccessful as juniors) will receive a different fixed wage and status compared to their successful colleagues of the same cohort. Also, in both systems, unproductive types will stay outside of the firm.

The timing for a cohort joining the organization at date $t$ is as follows.

$t=0$ : A new cohort of workers are offered contracts that include a junior status level, a fixed wage and an incentive wage for the first period. The firm also commits itself to a second-period contract which includes a rule for allocating status, fixed wages and incentive wages contingent on first-period performance. The firm also commits itself whether to provide an employment guarantee or to fire unsuccessful seniors and replace them by new juniors.

$t=0.5$ : Junior workers choose an effort level given all of the above.

$t=1$ : Outputs are observed, transfers occur and agents are promoted, retained or fired according to the terms of the contract. Agents can quit voluntarily. New juniors enter the firm.

$t=1.5$ : Senior workers choose an effort level according to their current monetary incentive and status. 


\section{$t=2$ : Outputs are observed, transfers occur, senior workers retire.}

As the outcome $q_{i t}$ which depends on effort at date $t$ is random, some agents will be successful and others not. Then an agent is characterized by its productivity $b$ or $g$, and by the fact that he is either a junior worker indexed 1 , or a senior worker with a history of high past performance, denoted $h$, or a history of low past performance, denoted $l$.

\section{Solution of the model}

\subsection{Outside option}

In each period agents can work as individual entrepreneurs outside the firm; agents have the same kind of productivity inside and outside the firm. The only difference is that they work individually and, therefore, they cannot change the status allocation in their one-person firm. Hence, they also face different incentives. By virtue of assumption A.1, in a one-person firm, the only feasible status allocation is constant. The value of $s=1$ is due to the normalization assumption that each worker is born with one unit of status. Note that we do not consider the highly visible entrepreneurs here who become principals of large firms but individuals working on their own in a one-person firm. The per-period utility of a self-employed is

$$
E U_{i t}=q+e_{i t} \Delta q-a_{i} \frac{e_{i t}^{2}}{2}
$$

A self-employed agent will choose the optimal effort level $\underline{e}_{i}=\frac{\Delta q}{a_{i}}$ for all $t$. The resulting expected utility is

$$
E U_{i t} \equiv \underline{U}_{i}=q+\frac{\Delta q^{2}}{2 a_{i}}
$$

Since $a_{b}>a_{g}$, reservation utility is type-dependent with $\underline{U}_{g}>\underline{U}_{b}$.

\subsection{Workers' optimal effort choices}

In the following, we assume that the firm is in steady state and consequently drop the time index. We consider first the problem of a senior worker at date 1.5. This problem is isomorphic in both promotion hierarchies, although the respective status allocations and wages differ. We can thus save on notation in terms of the type of the hierarchy considered.

Let $e_{i p}\left(s_{p}, w_{p}, \Delta w_{p}\right)$ denote the optimal effort level of a senior worker of type $i \in\{g, b\}$ with status $s_{p}$ and compensation $\left(w_{p}, \Delta w_{p}\right)$, where index $p=l$ stands for low and $h$ for high past performance. The agent maximizes the following programme:

$$
E U_{i p}=s_{p}\left(e_{i p} \Delta w_{p}+w_{p}\right)-a_{i} \frac{e_{i p}^{2}}{2} .
$$


The first-order condition (which is sufficient) implies:

$$
e_{i p}^{*}=\min \left\{\frac{s_{p} \Delta w_{p}}{a_{i}}, 1\right\}, p \in\{h, l\} .
$$

We consider next the problem of a junior worker of type $i$ maximizing his expected utility, at date $t=0.5$. Agent $i$ chooses his effort $e_{i 1}$ to solve:

$$
E U_{i 1}=s_{1}\left(e_{i 1} \Delta w_{1}+w_{1}\right)+e_{i 1} \Delta U_{i}-a_{i} \frac{e_{i 1}^{2}}{2} .
$$

Here $\Delta U_{i}=E U_{i h}-\underline{U}_{i}$ in an up-or-out system, and $\Delta U_{i}=E U_{i h}-E U_{i l}$ for standard promotion practices. The first-order condition (which is sufficient) implies:

$$
e_{i 1}^{*}=\min \left\{\frac{s_{1} \Delta w_{1}+\Delta U_{i}}{a_{i}}, 1\right\} .
$$

We will restrict the analysis to the meaningful case in which there is an interior solution with respect to effort. This can be guaranteed by assuming $a_{g}$ to be high and $\Delta q$ low enough so that in equilibrium $e_{i 1}<1$ and $e_{i p} \leq 1$ :

$$
a_{g} \geq 37.5 q \text { and } \Delta q \leq 5 q
$$

\subsection{Optimal incentive contracts without status differentiation}

We here describe a benchmark case in which the firm relies only on monetary incentives. Status allocation is constant and identical among workers so that $s_{i}=1 \forall i$. Consider a single period where the firm maximizes the expected profit function with respect to a fixed wage and bonus allocation. Assuming a proportion $x$ of good agents, the program is as follows:

$$
\max _{w_{i}, \Delta w_{i}} \Pi=x\left(q-w_{g}+e_{g}\left(\Delta q-\Delta w_{g}\right)\right)+(1-x)\left(q-w_{b}+e_{b}\left(\Delta q-\Delta w_{b}\right)\right)
$$

subject to

$$
\begin{aligned}
& w_{i} \geq 0, \\
& e_{i}=\min \left\{\frac{\Delta w_{i}}{a_{i}}, 1\right\}, \\
& E U_{i}=w_{i}+e_{i} \Delta w_{i}-a_{i} \frac{e_{i}^{2}}{2} \geq \underline{U}_{i}=q+\frac{\Delta q^{2}}{2 a_{i}}, i \in\{g, b\} .
\end{aligned}
$$

LL stands for limited liability, IC is the incentive compatibility, and IR the individual rationality constraint. In the program above we have implicitly assumed that the firm is able to sort out workers of type $b$ and $g$ at zero cost. 
We can readily show that the optimal solution derived under this assumption is implementable under the more realistic framework of asymmetric information. Under assumption A.2 we will get an interior solution for the effort so that $e_{i}=\frac{\Delta w_{i}}{a_{i}}$. The IR constraint then writes: $E U_{i}=w_{i}+\frac{\Delta w_{i}^{2}}{2 a_{i}} \geq q+\frac{\Delta q^{2}}{2 a_{i}}, i \in\{g, b\}$. The firm's objective function decreases with $w_{i}$ so that the IR constraints bind. Substituting $w_{i}=q+\frac{\Delta q^{2}}{2 a_{i}}-\frac{\Delta w_{i}^{2}}{2 a_{i}}$ in (8) and optimizing with respect to $\Delta w_{i}$ yields: $\Delta w_{i}=\Delta q$ so that $w_{i}=q \forall i \in\{g, b\}$. It is easy to check that under assumption A.2 effort is lower than 1. This solution is implementable under asymmetric information as workers become residual claimants of their work and the firm makes zero profit on both types of workers. Given agents' risk neutrality and the outside option where workers receive all of the surplus they produce, the firm cannot do better than this contract by postponing rewards. We next show that this result continues to hold in a dynamic context, the optimal dynamic solution is simply the replication of the optimal static solution. We focus on a steady-state solution.

Proposition 1 If status is not differentiated, the profit maximizing dynamic incentive contract is independent of the type or seniority of workers: $w=q$ and $\Delta w=\Delta q$. Both types of workers are entering the firm, and the firm makes zero profit.

The proofs of this and all following results are derived in the Appendix. Notice that Proposition 1 is in contrast to Ohlendorf and Schmitz (2012) who show that the optimal dynamic contract displays memory. The reason is that they impose the reservation utility to be zero, $\underline{U}=0$, implying that the individual rationality constraints of the workers never bind. Consequently, in Ohlendorf and Schmitz (2012) the binding constraint is always the limited liability (LL) one and workers are earning a rent. In a dynamic context, the principal can extract part of this rent by introducing memory in the contract.

In our model, as the workers' outside option depends on their productivity, their individual rationality constraint binds, and the principal cannot improve on the static solution. More generally and quite intuitively, the Ohlendorf and Schmitz (2012) memory result does not hold when the worker is not earning a rent, i.e., when the reservation utility is sufficiently high so that the individual rationality constraints of the workers bind. If the outside option for agents is to work as individual entrepreneurs, the firm cannot make any profits in a situation without status incentives. Consequently, in order for a firm to be able to earn profits, this must be owing to status considerations, as we show in what follows.

\subsection{Two types of promotion hierarchy}

We present two propositions describing the wages in the up-or-out system, and with standard promotion practices. We then investigate under what conditions either one or the other is optimal for the firm. While be build on the main result in Auriol and Renault (2008), namely that juniors receive zero wages and status, our interest is to investigate the precise shapes of the promotion hierarchy, and to predict under what circumstances either of the 
two promotion systems is optimal. We also show that a promotion hierarchy induces less productive types to stay outside of the firm (which increases profits).

In the up-or-out system, the firm maximizes expected output minus wages, subject to the ex ante participation constraint of the good worker, and the interim participation constraint of a successful worker to be satisfied. The firm also faces the status feasibility constraint (A.1) and limited liability constraints. The following Proposition fully characterizes the optimal up-or-out system.

Proposition 2 In an up-or-out system, unsuccessful former juniors must leave the firm, while successful juniors are promoted. The optimal up-or-out contract induces sorting, that is, only the good workers apply for jobs in the firm. Wages and status allocation are as follows: (i) junior workers receive minimum rewards and status $w_{1}^{U}=0, \Delta w_{1}^{U}=0, s_{1}^{U}=0$; (ii) successful seniors receive $w_{h}^{U}=\frac{2 \sqrt{a_{g} \underline{U}_{g}}}{s_{h}^{U}}-s_{h}^{U} \frac{\Delta q^{2}}{2 a_{g}}+\frac{\underline{U}_{g}}{s_{h}^{U}}, \Delta w_{h}^{U}=\Delta q, s_{h}^{U}=1+\frac{1}{2} \sqrt{\frac{a_{g}}{\underline{U}_{g}}}$.

The up-or-out hierarchy provides strong incentives to junior workers. By bundling social and material rewards in one state of the world, large firms create prizes for their workers who are willing to take the gamble to get the prestigious and lucrative promotion. They work harder than in self employment $\left(e_{1 g}^{U}=2 \sqrt{\frac{\underline{U}_{g}}{a_{g}}}=2 \sqrt{\frac{q}{a_{g}}+\frac{\Delta q^{2}}{2 a_{g}^{2}}}>\underline{e}_{g}=\frac{\Delta q}{a_{g}}\right)$ in the hope to stay on board and receive a substantial increase in status and high wages. The principal actually loses money on the promoted seniors, but this loss is more than compensated by the surplus he extracts from the juniors. A large firm that offers an up-or-out hierarchy engineers promotion packages that combine social and material rewards, and, through this packaging, makes profits.

A promotion hierarchy also makes it possible to sort out at no cost the most productive workers. Indeed, in up-or-out promotion systems social and monetary rewards are not only delayed in time, as in any promotion system, but they are allocated only to successful employees. With such a structure, workers with low ability are less likely to get a reward for their effort. They rather stick to self-employment. The ability to attract the most productive workers and to extract a high level of effort from them creates a competitive edge for large firms.

The program with standard promotion practices has one main difference compared to the up-or-out system: with standard promotion practices, nobody is fired. Unsuccessful juniors stay in the firm, but the firm differentiates status and wages of successful vs. unsuccessful seniors. The following proposition summarizes:

Proposition 3 With standard promotion practices, all workers stay in the firm. The optimal contract with standard promotion practices induces sorting, that is, only the good workers apply for jobs in the firm. Wages and status allocation are as follows: (i) junior workers re- 
ceive minimum rewards and status $\left(w_{1}^{S}=0, \Delta w_{1}^{S}=0, s_{1}^{S}=0\right)$; (ii) successful seniors receive

$$
w_{h}^{S}=w_{l}^{S} \sqrt{\sqrt{\frac{2 a_{g}}{\underline{U}_{g}}}+1}, \Delta w_{h}^{S}=\Delta q, s_{h}^{S}=\frac{2 \sqrt{\sqrt{\frac{2 a_{g}}{\underline{U}_{g}}}+1}}{1+\sqrt{\frac{2 \underline{U_{g}}}{a_{g}}}\left(\sqrt{\sqrt{\frac{2 a_{g}}{\underline{U}_{g}}}+1}-1\right)}
$$

and (iii) unsuccessful seniors receive

$$
w_{l}^{S}=\frac{\underline{U}_{g}}{s_{l}^{S}}-\frac{s_{l}^{S} \Delta q^{2}}{2 a_{g}}, \Delta w_{l}^{S}=\Delta q, s_{l}^{S}=\frac{2}{1+\sqrt{\frac{2 \underline{U_{g}}}{a_{g}}}\left(\sqrt{\sqrt{\frac{2 a_{g}}{\underline{U}_{g}}}+1}-1\right)} .
$$

Unsuccessful seniors receive a lower status compared to their successful counterparts. A firm with standard promotion practices loses money on both types of seniors; the loss is more than compensated by the surplus extracted from the juniors.

Both up-or-out and standard promotion induce sorting and in both promotion hierarchies, juniors only receive incentives linked to promotion. Seniors have first best incentives; they receive the entire surplus associated with a success. Comparing the profits of the firm, we can answer the question of optimality of the two systems in the next Proposition. The two threshold levels $\Delta^{l} \sim 2.07$ and $a^{l} \sim 182.95$ are derived in the proof.

Proposition 4 A firm's optimal choice between up-or-out or standard promotion practices depends on the payoff associated to a success $\Delta q$, and the difficulty of achieving a success for the productive workers $a_{g}$ : (i) if $\Delta q$ is sufficiently large compared to $q\left(\frac{\Delta q}{q} \geq \Delta^{l}\right)$, then, up-or-out is optimal; (ii) if $\frac{\Delta q}{q}<\Delta^{l}$ and $a_{g}$ sufficiently large compared to $q\left(\frac{a_{g}}{q} \geq a^{l}\right)$, then, again up-or-out is optimal; (iii) otherwise, standard promotion practices are optimal.

To understand the intuition for the result, consider first a one-period setting. If effort levels were contractible, the firm would like to make the most of the complementarity between status and income and concentrate both on one sole worker. The firm would then offer each agent the participation in a lottery where only one winner receives positive status and income. However, status is assigned at the beginning of the period when each worker is given his initial position in the firm's hierarchy. Thus, if effort is not contractible, a worker who has received zero status cannot be induced to work. With moral hazard, a lottery solution is therefore not optimal. The firm could, however, still differentiate status among workers to some extent. Giving some agents more status than others would induce the former to work more. But because the cost of effort is convex, differentiation does not pay off in a one-period setting and the firm optimally gives the same status and compensation scheme to each worker (Auriol and Renault, 2008, Proposition 2). Without status redistribution, the firm cannot make positive profits in a one-period setting. The case of senior workers in the second period of our model who will retire at the end of the period corresponds to this one-period situation. Differentiation thus comes at a cost and can only be optimal when it 
induces the juniors to work harder in the first period. This logic also applies to a dynamic setting in which the principal has no possibility to commit him or herself to contractual arrangements.

In a dynamic setting with contractual commitment, other allocations of status are optimal. Consider our two-period setting. Workers are not earning a rent in our model and thus juniors inside the firm receive the same expected utility as if they were working for two periods in self-employment. However, the intergenerational redistribution of status allows the firm to make positive profits. By bundling high status and high income in the same state of nature, it exploits the complementarity between status and income in workers' preferences and lowers the total wage bill. Wages and status are shifted to the second period of the professional life to provide the workers with incentives to work, explaining the backloaded structure of the dynamic incentive scheme.

Given the backloaded structure, the choice between both hierarchies builds on the distinct advantages of each of them: At first glance, it would seem that up-or-out is always preferable because it offers steeper incentives to juniors. This is indeed one of the main advantages of an up-or-out system. In addition, the firm saves on the payment for the unsuccessful seniors who have to leave the firm and work self-employed. Taken together, when incentives should be strong because success is important ( $\Delta q$ is sufficiently large) or hard to achieve ( $a_{g}$ sufficiently large compared to $q$ ), up-or-out is optimal.

However, the problem with an up-or-out hierarchy is that the "loser prize" for the unsuccessful seniors cannot be adjusted by the firm. In particular, in our model no status can be transferred to or from the individuals who have to leave. Thus, consider a situation where incentives are of little importance because a low effort is optimal (either because achieving a high output is easy or because $\Delta q$ is low). Then, a firm with standard promotion practices can make the most of the complementarity between status and income by giving all juniors no status and a zero wage and distributing status and income fairly evenly between all seniors with only a low differentiation based on success (i.e., by implementing some kind of promotion by seniority system). In contrast, because of the low prize for the losers in the up-or-out system, the firm would need to give a higher status and income to successful seniors to fulfill the participation constraint. Juniors would then spend more effort than needed which has to be compensated by the firm. Therefore, when incentives to juniors are less important, standard promotion practices are preferable.

\section{Implications and discussion}

The last Proposition establishes that the up-or-out system dominates when the surplus generated by high effort is sufficiently large and whenever the difficulty of achieving a success is sufficiently large. This is in line with casual observations that up-or-out dominates in fields in which there are large sums at stake or in which people have to work very hard to succeed. This is also in line with the findings in Levin and Tadelis (2005) on partnerships and upor-out contracts that prevail when success (or quality) is of high importance. Examples include law firms or consulting firms in which winning a law suit or a new, important client 
makes all the difference and people work both intensively and extensively, in particular when fighting for partnership status. But this also is in line with the arts, as for example in the music business where the "up" consists in a long-term contract with a publishing house, or in science where the "up" is getting tenure. In many (but not all) countries scientists are used to be granted tenure only after six to ten years on the job, and not making tenure at a prestigious university is perceived as a substantial loss in expected status.

There are a number of observations and predictions. The first relates to the wage and status profiles over time. Juniors who enter the up-or-out system have a higher variance in terms of both status and wages than with standard promotion practices: successful seniors are promoted to jobs with high wages and high status, while unsuccessful seniors leave to self-employment. With standard promotion practices both wage and status profiles are less steep than under up-or-out. Most importantly, unsuccessful seniors stay in the firm and enjoy positive status. Nonetheless, in both systems, juniors are paid minimum wages and receive no status.

A second observation builds on a comparison of effort levels of juniors. Omitting the subscript for the type of worker, because only $g$ type workers enter the firm, this is $e_{1}^{U}=$ $2 \sqrt{\frac{\underline{U}_{g}}{a_{g}}}$ in up-or-out, compared to $e_{1}^{S}=\sqrt{\frac{2 \underline{U}_{g}}{a_{g}}}$ with standard promotion practices. Thus, we have $e_{1}^{S}=\frac{1}{\sqrt{2}} e_{1}^{U}$; hence juniors work harder under an up-or-out contract. This seems to be in line with casual evidence, whereby junior employees in industries that use up-or-out contracts such as consulting, law, or investment banking, are reputed to work harder than junior employees in industries that use standard promotion practices.

Third, effort of seniors is $e_{h}^{U}=s_{h}^{U} \frac{\Delta q}{a_{g}}$ with up-or-out and $e_{h}^{S}=s_{h}^{S} \frac{\Delta q}{a_{g}}$ or $e_{l}^{S}=s_{l}^{S} \frac{\Delta q}{a_{g}}$ with standard promotion practices. We have $1 \leq s_{l}^{S} \leq 2 \leq s_{h}^{S}$. Therefore, successful seniors work harder than unsuccessful seniors with standard promotion practices. However, for the high performers of the past in the two systems, the comparison is not as clear cut. We can have $s_{h}^{U} \gtreqless s_{h}^{S}$. Therefore, seniors in the top position of a firm with standard promotion practices (e.g. c-level executives in large industrial companies) may work harder than seniors in a firm that employs up-or-out contracts.

A fourth prediction applies to the structure of the hierarchy in terms of the ratio between juniors and seniors, the span of control. As juniors work harder in an up-or-out hierarchy, there are more successful seniors than with standard promotion practices, but not everyone succeeds. Unsuccessful seniors are replaced by new juniors, while for standard promotion practices, the unsuccessful seniors stay on. As a consequence, the ratio of juniors and unsuccessful seniors over successful seniors with standard promotion practices is larger than the ratio of juniors over seniors in an up-or-out organization. A Web Appendix contains an illustration of this prediction employing data from U.S. and French economics departments.

Our model can also at least partially rationalize some of the transformations of the large law firms that over the last decades have changed their promotion systems away from pure up-or-out contracts. Many of them now also employ permanent lawyers who are not partners (Galanter and Palay, 1991) and thus have moved toward standard promotion practices. Gorman (1999) discusses three factors that lead to this change and examines these factors 
empirically. Ghosh and Waldman (2010) show that their model is consistent with two of these factors. In particular, an increase in the complexity of legal work in the 1980s has led to an increase in the required firm specific human capital. As we will argue below, the resulting shift to standard promotion practices is also in line with our model. ${ }^{11}$ Our model is also in line with the third factor examined by Gorman (1999): a shift in values. In particular, she argues that, traditionally, collegiality norms have played an important role in large law firms, implying that all senior employees should be "equal in status" (Gorman, 1999, p. 646). A shift in values challenged this equality norm and paved the way for standard promotion practices with status differentiation among seniors. This is consistent with the main interest of our theory that status concerns are an important driver in the choice of promotion system. More specifically, ruling out status differentiation between senior workers would in our model also favor up-or-out contracts. The reason is that on the one hand wage differentiation between workers of equal status is not optimal because of the complementarity between status and income, and on the other hand without any differentiation juniors cannot be induced to work. Thus, if all seniors are required to have the same status, up-or-out contracts can fulfill this restriction while still giving high incentives to juniors.

We would like to conclude with a remark about the impact of firm-specific human capital on the optimal promotion system. While the model of Kahn and Huberman (1988) predicts that up-or-out should prevail when firm-specific human capital is important, the model of Ghosh and Waldman (2010) predicts the opposite. Introducing human capital in our framework would mean that seniors become more efficient over time because they acquire human capital. The acquisition of human capital does not affect successful seniors who will always work for the firm, and a fortiori do so when human capital is specific to the firm. However, human capital acquisition could shift the optimality of up-or-out versus standard promotion practices, because in up-or-out, the firm would lose productive senior workers and would need to replace them by less productive juniors. Put differently, under standard promotion practices, less useful firm-specific capital is lost for reasons of providing steep incentives. Depending on the importance of human capital for production, up-or-out could altogether vanish. In any case, the threshold levels (both in terms of the difficulty of achieving and of the returns to a success) would shift, making standard promotion practices more likely. This is in line with the theoretical results in Ghosh and Waldman (2010) and with empirical evidence in Gorman (1999).

\section{Concluding remarks}

We have suggested a simple dynamic agency model in which firms can make profits by offering promotion opportunities to successful juniors. The hierarchies differ with respect

\footnotetext{
${ }^{11}$ The second factor discussed in Ghosh and Waldman (2010) is that client relationships have become less important leading to low- and high-level jobs in law firms becoming less similar. Given that in our model juniors and seniors conduct the same type of work, our model cannot account for this factor.
} 
to the treatment of unsuccessful juniors. In up-or-out, these unsuccessful juniors must go, while with standard promotion practices they can stay. We have shown that these promotion hierarchies both succeed in inducing sorting: only the more productive workers want to work in these organizations. We have shown that up-or-out is the profit maximizing promotion hierarchy for very high payoffs of successful work of juniors and when the task is causing high costs of effort, while otherwise standard promotion practices dominate. Finally, we have derived some testable implications. A Web Appendix presents some descriptive data from the French and the U.S. university system in line with our theory. 


\section{References}

Auriol, Emmanuelle and Régis Renault (2001), "Incentive Hierarchies", Annales d'Economie et de Statstique, 63-64, 261-82

Auriol, Emmanuelle and Régis Renault (2008), "Status and Incentives", RAND Journal of Economics, 39(1), 305-326

Baker, George, Michael Gibbs and Bengt Holmström (1994), "The Internal Economics of the Firm", Quarterly Journal of Economics, 109(4), 881-919

Ball, Sheryl and Catherine Eckel (1998), "The Economic Value of Status", Journal of Socio-economics, 27(4), 495-514.

Ball, Sheryl, Catherine Eckel, Philip J. Grossman and William Zame (2001), "Status in Markets", Quarterly Journal of Economics, 116(1), 161-188.

Bar-Isaac, Heski (2007), "Something to Prove: Reputation in Teams." The RAND Journal of Economics, 38(2), 495-511

Barnard, Chester (1938): The Functions of the Executive, Harvard University Press, Cambridge (Mass.)

Besley, Tim and Maitreesh Ghatak (2008), "Status Incentives", American Economic Review (Papers and Proceedings), 98(2), 206-211

Bosquet, Clément, Pierre-Philippe Combes and Laurent Linnemer (2010), "La publication d'articles de recherche en économie en France en 2008: Disparités actuelles et évolutions depuis 1998", Rapport pour la Direction Générale de la Recherche et de l'Innovation (DGRI) du Ministère de l'Enseignement Supérieur et de la Recherche

Bradler, Christiane and Susanne Neckermann (2011), "Motivational Production Function - A Field Experiment", paper presented in the workshop on "Field Experiments in Organizational and Labor Economics", Frankfurt.

Centers, Richard and Daphne E. Bugental (1966), "Extrinsic and Intrinsic Job Satisfaction Among Different Segments of Work Population", Journal of Applied Psychology, 50(3), 193-197

Demougin, Dominique and Aloysius Siow (1994), "Careers in Ongoing Organizations", American Economic Review, 84(5), 1261-1277

Doeringer, Peter and Michael Piore (1971), Internal Labor markets and Manpower Analysis, Heath Lexington Books, D.C. Heath and Company Lexington, Massachusetts

Drucker, Peter F. (1954), The Practice of Management, Harper \& Row, New York 
Dusansky, Richard and Clayton J. Vernon (1998), "Rankings of U.S. Economics Departments", Journal of Economic Perspectives, 12(1), 157-170

Frank, Robert (1985), Choosing the Right Pond: Human Behavior and the Quest for Status, Oxford University Press

Garicano, Luis (2000), "Hierarchies and the Organization of Knowledge in Production", Journal of Political Economy, 108(5), 874-904.

Ghosh, Suman and Michael Waldman (2010), "Standard Promotion Practices versus Upor-Out Contracts", RAND Journal of Economics, 41(2), 301-325

Gibbons, Robert and Michael Waldman (1999), "Careers in Organizations: Theory and Evidence", Handbook of Labor Economics, Vol. 3B, 2373-2438, Elsevier

Huberman, Bernardo A., Christoph H. Loch and Ayse Önçüler (2004), "Status As a Valued Resource", Social Psychology Quarterly, 67(1), 103-114

Jemmott, John B. and Elida Gonzalez (1989), "Social Status, the Status Distribution, and Performance in Small Groups1", Journal of Applied Social Psychology, 19(7), 584-598.

Kahn, Charles and Gur Huberman (1988), "Two-sided Uncertainty and "Up-or-Out" Contracts", Journal of Labor Economics, 6(4), 423-444

Lazear, Edward (2000) "The Power of Incentives", American Economic Review, 90(2), 410-414.

Lazear, Edward and Sherwin Rosen (1981), "Rank-order Tournaments as Optimum Labor Contracts", Journal of Political Economy 89(5), 841-864

Levin, Jonathan and Steven Tadelis (2005) "Profit Sharing and the Role of Professional Partnerships." The Quarterly Journal of Economics, 120(1), 131-171

Malcomson, James (1984), "Work Incentives, Hierarchy and Internal Labor Markets", Journal of Political Economy 92, 486-507

Marino, Anthony and Oguzhan Ozbas (2011) "Disclosure of Status in an Agency Setting." Working Paper

Maslow, Abraham (1954), "Motivation and Personality", New York, Harper and Row

Neckermann, Susanne and Michael Kosfeld (2011). Getting more work for nothing? Symbolic Awards and Worker Perfomance. American Economic Journal. Microeconomics, 3, $1-16$.

Moldovanu, Benny, Aner Sela and Xianwen Shi (2007), "Contests for Status", Journal of Political Economy, 115(2), 338-363 
O'Flaherty, Brendan and Aloysius Siow (1995), "Up-or-Out Rules in the Market for Lawyers", Journal of Labor Economics, 13(4), 709-735

Ohlendorf, Susanne and Patrick W. Schmitz (2012), "Repeated Moral Hazard and Contracts with Memory: The Case of Risk-Neutrality", International Economic Review, 53(2), 433-452

Prendergast, Canice (1993), "The Role of Promotion in Inducing Specific Human Capital Acquisition", Quarterly Journal of Economics, 108(2), 523-534

Smeets, Valerie, Michael Waldman and Frederic Warzynski (2013), "Performance, Career Dynamics, and Span of Control", DP Cornell, (ICS 2013-001).

Stajkovic, Alexander and Fred Luthans (2003), "Behavioral Management and Task Performance in Organizations: Conceptual Background, Meta-Analysis, and Test of Alternative Models, Personnel Psychology, 56(1), 155-194

von Siemens, Ferdinand A. (2010), "Heterogeneous Social Preferences, Screening, and Employment Contracts", Oxford Economic Papers, 63(3), 499-522

Waldman, Michael (1984), "Job Assignments, Signalling and Efficiency", Rand Journal of Economics, 15(2), 255-267

Waldman, Michael (1990), "Up-Or-Out Contracts: A Signaling Perspective", Journal of Labor Economics, 8(2), 230-250

Waldman, Michael (2009), "Theory and Evidence in Internal Labor Markets", forthcoming in Robert Gibbons and John Roberts (eds.), Handbook of Organizational Economics. 


\section{Appendix Proof of Proposition 1}

We focus on a steady-state solution so that the contracts are independent of time. Moreover, we first neglect the problem of asymmetric information, assuming that the principal can observe workers' types. We then show that the solution obtained under this assumption is implementable under the more realistic framework of asymmetric information. Let $\Delta U_{i}=E U_{i h}-E U_{i l}$. When she is not differentiating status, under complete information about workers types, the principal optimizes independently for each type of agent $i=g, b$ the following objective function:

$$
\begin{aligned}
& \max \Pi=q-w_{i 1}+e_{i 1}\left(\Delta q-\Delta w_{i 1}\right)+e_{i 1}\left(q-w_{i h}+e_{i h}\left(\Delta q-\Delta w_{i h}\right)\right)+\left(1-e_{i 1}\right)\left(q-w_{i l}+e_{i l}\left(\Delta q-\Delta w_{i l}\right)\right) \\
& \text { s.t. } \quad \begin{aligned}
w_{i k} & \geq 0, \quad k=1, h, l \\
e_{i k} & =\min \left\{\frac{\Delta w_{i k}}{a_{i}}, 1\right\} \quad k=h, l \quad e_{i 1}=\min \left\{\frac{\Delta w_{i 1}+\Delta U_{i}}{a_{i}}, 1\right\} \quad \text { (ICk) } \\
E U_{i k} & =w_{i k}+e_{i k} \Delta w_{i k}-a_{i} \frac{e_{i k}^{2}}{2} \geq \underline{U}_{i}=q+\frac{\Delta q^{2}}{2 a_{i}} \quad k=h, l . \\
E U_{i 1} & =w_{i 1}+e_{i 1}\left(\Delta w_{i 1}+\Delta U_{i}\right)-a_{i} \frac{e_{i 1}^{2}}{2} \geq 2 \underline{U}_{i}=2 q+\frac{\Delta q^{2}}{a_{i}}
\end{aligned}
\end{aligned}
$$

We focus on an interior solution for effort. We will check later that the optimal contract leads to interior solutions under assumption A.2. Substituting $e_{i k}=\frac{\Delta w_{i k}}{a_{i}}$ and $e_{i 1}=\frac{\Delta w_{i 1}+\Delta U_{i}}{a_{i}}$ in $(\mathrm{IRk})$ and (IR1) yields: $E U_{i k}=w_{i k}+\frac{\Delta w_{i k}^{2}}{2 a_{i}} \geq \underline{U}_{i}=q+\frac{\Delta q^{2}}{2 a_{i}}$ for $k=h, l$ and $E U_{i 1}=$ $w_{i 1}+\frac{\left(\Delta w_{i 1}+\Delta U_{i}\right)^{2}}{2 a_{i}} \geq 2 \underline{U}_{i}=2 q+\frac{\Delta q^{2}}{a_{i}}$. The principal's objective function decreases with the rent she leaves to the workers. The only reason she mights leave a rent to senior workers is to provide incentives for junior workers. What matters for juniors' incentives is the difference in utility they will receive in the future depending on whether they succeed or fail: $\Delta U_{i}=$ $E U_{i h}-E U_{i l}$. It is thus optimal to minimize payment to previously unsuccessful workers: $E U_{i l}=w_{i l}+\frac{\Delta w_{i l}^{2}}{2 a_{i}}=q+\frac{\Delta q^{2}}{2 a_{i}}$. We deduce that $w_{i l}=q+\frac{\Delta q^{2}}{2 a_{i}}-\frac{\Delta w_{i l}^{2}}{2 a_{i}}$. Similarly we have $E U_{i h}=\Delta U_{i}+E U_{i l}$ so that $w_{i h}=\Delta U_{i}+q+\frac{\Delta q^{2}}{2 a_{i}}-\frac{\Delta w_{i h}^{2}}{2 a_{i}}$. Finally we deduce from (IR1) that $w_{i 1}=2 q+\frac{\Delta q^{2}}{a_{i}}-\frac{\left(\Delta w_{i 1}+\Delta U_{i}\right)^{2}}{2 a_{i}}$. The principal optimizes

$\Pi=2 q-w_{i 1}+e_{i 1}\left(\Delta q-\Delta w_{i 1}-\left(w_{i h}-w_{i l}\right)+e_{i h}\left(\Delta q-\Delta w_{i h}\right)\right)+\left(1-e_{i 1}\right) e_{i l}\left(\Delta q-\Delta w_{i l}\right)-w_{i l}$.

Substituting $w_{i 1}, w_{i l}, w_{i h}-w_{i l}=\Delta U_{i}-\frac{\Delta w_{i h}^{2}-\Delta w_{i l}^{2}}{2 a_{i}}, e_{i 1}$ and $e_{i k}$ for $k=h$, l, yields:

$\Pi=-q-\frac{\Delta q^{2}}{a_{i}}-\frac{\left(\Delta q-\left(\Delta w_{i 1}+\Delta U_{i}\right)\right)^{2}}{2 a_{i}}+\frac{\Delta w_{i 1}+\Delta U_{i}}{a_{i}} \frac{\Delta w_{i h}}{2 a_{i}}\left(2 \Delta q-\Delta w_{i h}\right)+\left(1-\frac{\Delta w_{i 1}+\Delta U_{i}}{a_{i}}\right) \frac{\Delta w_{i l}}{2 a_{i}}\left(2 \Delta q-\Delta w_{i l}\right)$

One can readily see that $\Delta w_{i 1}$ and $\Delta U_{i}$ are perfect substitutes in the principal objective function. Optimizing the objective function with respect to $\Delta w_{i k}$ yields the FOC with $2 \Delta q-2 \Delta w_{i k}=0$ for $k=h, l$. Under our assumptions it is easy to check that the objective 
function $\Pi$ is concave in $\Delta w_{i h}$ and $\Delta w_{i l}$ for all $\Delta w_{i 1}+\Delta U_{i} \geq 0$. We deduce that at the optimum $\Delta w_{i h}=\Delta w_{i l}=\Delta q$. The principal optimizes next with respect to $\Delta W_{i}=$ $\Delta w_{i 1}+\Delta U_{i}$ the function $\Pi=-q-\frac{\Delta q^{2}}{2 a_{i}}-\frac{\left(\Delta q-\Delta W_{i}\right)^{2}}{2 a_{i}}$. We deduce that $\Delta W_{i}=\Delta w_{i 1}+\Delta U_{i}=\Delta q$. At the optimum the principal is indifferent in providing incentives to junior workers through the bonus $\Delta w_{i 1}=\Delta q$ or through the difference in fixed wages between previously successful and unsuccessful senior workers $\Delta U_{i}=w_{i h}-w_{i l}=\Delta q$. These two ways of motivating the workers are perfect substitutes. Finally, we need to check that the solution satisfies $e_{i k} \leq 1$ and $e_{i 1} \leq 1$. Both conditions are equivalent to $\Delta q \leq a_{i}$. Since $a_{g}<a_{b}$, this is fulfilled given assumption A.2. QED

\section{Proof of Proposition 2}

We first show that it is optimal for juniors to receive minimal wages and status: $\Delta w_{1}^{U}=$ $w_{1}^{U}=s_{1}^{U}=0$. The proof is adapted from the proof of the first part of Proposition 3 in Auriol and Renault (2008). We cannot apply it directly here as conditions A.1, A.2, and A.3 in Auriol and Renault (2008) are not fully holding in the case we study here. Moreover, Auriol and Renault $(2001,2008)$ focus on standard promotion practices and rule out up-orout incentive schemes. This changes the proof. We show below that if the firm chooses to offer different contracts to different types of workers, these contracts will be such that juniors receive minimal wages and status. We avoid introducing additional notation by neglecting here the index for possible different types of workers.

If $s_{1}^{U}=0$, then it is optimal to set $w_{1}^{U}=\Delta w_{1}^{U}=0$. Thus we need to show that $s_{1}^{U}=0$. Suppose to the contrary that $s_{1}^{U}>0$. At some date $t$ the principal may switch to a new contract where the first term represents status, the second fixed wage, and the third bonus:

$$
c_{1}^{U^{\prime}}=(0,0,0), c_{h}^{U \prime}=\left(s_{h}^{U}+\frac{s_{1}^{U}}{\mu\left(e_{1}^{U}\right)}, \frac{s_{h}^{U} w_{h}^{U}+\frac{s_{1}^{U}}{\mu\left(e_{1}^{U}\right)}\left(w_{1}^{U}+\mu\left(e_{1}^{U}\right) \Delta w_{1}^{U}\right)}{s_{h}^{U}+\frac{s_{1}^{U}}{\mu\left(e_{1}^{U}\right)}}, \frac{s_{h}^{U} \Delta w_{h}^{U}}{s_{h}^{U}+\frac{s_{1}^{U}}{\mu\left(e_{1}^{U}\right)}}\right) .
$$

If each generation from $t$ on is offered these contracts, by construction whatever the worker's type the youngs' expected intertemporal utility is held constant. Basically, the youngs' wages are transferred from the first to the second period while being divided by the ratio of the original period 1 status to the new second period status $\frac{s_{1}^{U}}{\frac{s_{1}^{U}}{\mu\left(e_{1}^{U}\right)}+s_{h}^{U}}$, so that the increase in status exactly compensates for the decrease in income. On the other hand, the utility of an old successful agent is increased by $s_{1}^{U}\left(\frac{w_{1}^{U}}{\mu\left(e_{1}^{U}\right)}+\Delta w_{1}^{U}\right)$. The utility of the old unsuccessful agents is unchanged as it is their reservation utility. Furthermore, as the overall incentives do not change, all effort levels are maintained. Let $E w_{1}^{U}=w_{1}^{U}+\mu\left(e_{1}^{U}\right) \Delta w_{1}^{U}$ and $E w_{h}^{U}=w_{h}^{U}+\mu\left(e_{h}^{U}\right) \Delta w_{h}^{U}$ be the expected wage bill for junior and senior workers in the original contract and $E w_{1}^{U^{\prime}}=0, E w_{h}^{U^{\prime}}=\frac{\frac{s_{1}^{U}}{\mu\left(e_{1}^{U}\right)}}{s_{h}^{U}+\frac{s_{1}^{U}}{\mu\left(e_{1}^{U}\right)}} E w_{1}^{U}+\frac{s_{h}^{U}}{s_{h}^{U}+\frac{s_{1}^{U}}{\mu\left(e_{1}^{U}\right)}} E w_{h}^{U}$ in the new contract. 
It is straightforward to check that the intertemporal wage bill for each generation is lower: that is $\mu\left(e_{1}^{U}\right) \frac{\frac{s_{1}^{U}}{\mu\left(e_{1}^{U}\right)}}{s_{h}^{U}+\frac{s_{1}^{U}}{\mu\left(e_{1}^{U}\right)}} E w_{1}^{U}+\frac{s_{h}^{U}}{s_{h}^{U}+\frac{s_{1}^{U}}{\mu\left(e_{1}^{U}\right)}} \mu\left(e_{1}^{U}\right) E w_{h}^{U}<E w_{1}^{U}+\mu\left(e_{1}^{U}\right) E w_{h}^{U}$. Hence, a steady state with $s_{1}^{U}>0$ cannot be part of any optimal solution of up-or-out contracts.

Thus, juniors receive minimal wages and status. If A.2 holds, $a_{g}$ is such that in equilibrium $e_{g 1}^{U}<1$ (i.e., $\Delta U_{g}<a_{g}$ ). The following Lemma establishes that $e_{g h}^{U}=\frac{s_{h}^{U} \Delta w_{h}^{U}}{a_{g}} \leq 1$.

Lemma 1 Without loss of generality, at the optimum: $s_{h}^{U} \Delta w_{h}^{U} \leq a_{g}$.

Proof. If $\Delta w_{h}^{U}>\frac{a_{g}}{s_{h}^{U}}$, then $e_{g h}^{U}=1$. Let $\epsilon=\Delta w_{h}^{U}-\frac{a_{g}}{s_{h}^{U}}$ and $\Delta w_{h}^{U \prime}=\Delta w_{h}^{U}-\epsilon$, and $w_{h}^{U \prime}=w_{h}^{U}+\epsilon$. This implies $e_{g h}^{U \prime}=e_{g h}^{U}=1, E U_{h}=E U_{h}^{\prime}$, and $E \Pi=E \Pi^{\prime}$.

By Lemma $1 e_{i h}^{U}=\frac{s_{h}^{U} \Delta w_{h}^{U}}{a_{i}}$, and by assumption $e_{i 1}^{U}=\frac{\Delta U_{i}}{a_{i}}<1$. This implies that $E U_{i h}=$ $s_{h}^{U}\left(e_{i h}^{U} \Delta w_{h}^{U}+w_{h}^{U}\right)-a_{i} \frac{e_{i h}^{U 2}}{2}=\frac{\left(s_{h}^{U} \Delta w_{h}^{U}\right)^{2}}{2 a_{i}}+s_{h}^{U} w_{h}^{U}$ and $E U_{i 1}=e_{i 1}^{U} \Delta U_{i}-a_{i} \frac{e_{1}^{U 2}}{2}=\frac{\Delta U_{i}^{2}}{2 a_{i}}$ with $\Delta U_{i}=$ $E U_{i h}-\underline{U}_{i}=\frac{\left(s_{h}^{U} \Delta w_{h}^{U}\right)^{2}}{2 a_{i}}+s_{h}^{U} w_{h}^{U}-\underline{U}_{i}$. Then $\frac{\partial \Delta U_{i}}{\partial w_{h}^{U}}=s_{h}^{U} ; \frac{\partial \Delta U_{i}}{\partial \Delta w_{h}^{U}}=\frac{s_{h}^{2} \Delta w_{h}^{U}}{a_{i}} ; \frac{\partial \Delta U_{i}}{\partial s_{h}^{U}}=\frac{s_{h}^{U} \Delta w_{h}^{U 2}}{a_{i}}+w_{h}^{U} ;$ and $\left(\frac{2 \Delta U_{g}}{a_{g}+\Delta U_{g}}\right)^{\prime}=\frac{2 a_{g} \Delta U_{g}^{\prime}}{\left(a_{g}+\Delta U_{g}\right)^{2}}$.

The firm maximizes expected profits:

$$
\begin{aligned}
\max E \Pi & =\frac{2}{1+e_{g 1}^{U}}\left(e_{g 1}^{U} \Delta q+q\right)+\frac{2 e_{g 1}^{U}}{1+e_{g 1}^{U}}\left(e_{g h}^{U}\left(\Delta q-\Delta w_{h}^{U}\right)-w_{h}^{U}+q\right) \\
& =2\left(\frac{e_{g 1}^{U}}{1+e_{g 1}^{U}}\left(\Delta q+e_{g h}^{U}\left(\Delta q-\Delta w_{h}^{U}\right)-w_{h}^{U}\right)+q\right)
\end{aligned}
$$

subject to $E U_{g h} \geq \underline{U}_{g}\left(I R_{g h}\right), E U_{g 1}=\frac{\Delta U_{g}^{2}}{2 a_{g}} \geq 2 \underline{U}_{g}\left(I R_{g 1}\right), E U_{b 1}=\frac{\Delta U_{b}^{2}}{2 a_{b}}<2 \underline{U}_{b}\left(I R_{b 1}\right)$, $\frac{2 e_{g 1}^{U}}{1+e_{g 1}^{U}} s_{h}^{U}=2$ (status feasibility), $w_{h}^{U} \geq 0(L L), e_{g 1}^{U}=\frac{\Delta U_{g}}{a_{g}}\left(I C_{g 1}\right), e_{g h}^{U}=\frac{s_{h}^{U} \Delta w_{h}^{U}}{a_{g}}\left(I C_{g h}\right)$ and $s_{h}^{U} \Delta w_{h}^{U} \leq a_{g}$. The Lagrangian is:

$$
\begin{aligned}
& L=\frac{2 \Delta U_{g}}{a_{g}+\Delta U_{g}}\left(\Delta q+\frac{s_{h}^{U} \Delta w_{h}^{U}}{a_{g}}\left(\Delta q-\Delta w_{h}^{U}\right)-w_{h}^{U}\right)+2 q+\alpha\left(\frac{\left(s_{h}^{U} \Delta w_{h}^{U}\right)^{2}}{2 a_{g}}+s_{h}^{U} w_{h}^{U}-\underline{U}_{g}\right) \\
& +\beta_{g}\left(\frac{\Delta U_{g}^{2}}{2 a_{g}}-2 \underline{U}_{g}\right)+\beta_{b}\left(2 \underline{U}_{b}-\frac{\Delta U_{b}^{2}}{2 a_{b}}\right)+\gamma\left(2-\frac{2 \Delta U_{g}}{a_{g}+\Delta U_{g}} s_{h}^{U}\right)+\lambda_{h} w_{h}^{U}+\epsilon_{h}\left(a_{g}-s_{h}^{U} \Delta w_{h}^{U}\right)
\end{aligned}
$$

Define $D:=\Delta q+\frac{s_{h}^{U} \Delta w_{h}^{U}}{a_{g}}\left(\Delta q-\Delta w_{h}^{U}\right)-w_{h}^{U}-\gamma s_{h}^{U}$ and $B:=\frac{2 D a_{g}}{\left(a_{g}+\Delta U_{g}\right)^{2}}+\beta_{g} \frac{\Delta U_{g}}{a_{g}}+\alpha$. From the Lagrangian, we derive the following conditions:

$$
\alpha \geq 0 ; \beta_{g} \geq 0 ; \beta_{b} \geq 0 ; \gamma \geq 0 ; \lambda_{h} \geq 0 ; \epsilon_{h} \geq 0
$$




$$
\begin{aligned}
& \alpha\left(E U_{g h}-\underline{U}_{g}\right)=0 \\
& \beta_{g}\left(\frac{\Delta U_{g}^{2}}{2 a_{g}}-2 \underline{U}_{g}\right)=0 \\
& \beta_{b}\left(2 \underline{U}_{b}-\frac{\Delta U_{b}^{2}}{2 a_{b}}\right)=0 \\
& \gamma\left(2-\frac{2 \Delta U_{g}}{a_{g}+\Delta U_{g}} s_{h}^{U}\right)=0 \\
& \lambda_{h} w_{h}^{U}=0 \\
& \epsilon_{h}\left(a_{g}-s_{h}^{U} \Delta w_{h}^{U}\right)=0 \\
& \frac{\partial L}{\partial \Delta w_{h}^{U}}=\frac{s_{h}^{U} \Delta w_{h}^{U}}{a_{g}} B-\frac{s_{h}^{U} \Delta w_{h}^{U}}{a_{b}^{2}} \beta_{b} \Delta U_{b}+\frac{2 \Delta U_{g}}{\left(a_{g}+\Delta U_{g}\right) a_{g}}\left(\Delta q-2 \Delta w_{h}^{U}\right)-\epsilon_{h}=0 \\
& \frac{\partial L}{\partial s_{h}^{U}}=\left(\frac{s_{h}^{U} \Delta w_{h}^{U}}{a_{g}}+\frac{w_{h}^{U}}{\Delta w_{h}^{U}}\right) B-\left(\frac{s_{h}^{U} \Delta w_{h}^{U}}{a_{b}}+\frac{w_{h}^{U}}{\Delta w_{h}^{U}}\right) \beta_{b} \frac{\Delta U_{b}}{a_{b}}+ \\
& \frac{\partial L}{a_{g}\left(a_{g}+\Delta U_{g}\right)}\left(\Delta q-\Delta w_{h}^{U}\right)-\gamma \frac{2 \Delta U_{g}}{\Delta w_{h}^{U}\left(a_{g}+\Delta U_{g}\right)}-\epsilon_{h}=0 \\
& \frac{\partial w_{h}^{U}}{U_{h}}=s_{h}^{U}\left(B-\beta_{b} \frac{\Delta U_{b}}{a_{b}}\right)-\frac{2 \Delta U_{g}}{a_{g}+\Delta U_{g}}+\lambda_{h}=0
\end{aligned}
$$

Depending on parameters different solutions can occur. We focus on the case where in equilibrium:

$$
\begin{aligned}
& w_{h}^{U}>0 \text { which implies } \lambda_{h}=0 \\
& \beta_{b}=0 \text { which implies } E U_{b 1}<2 \underline{U}_{b} .
\end{aligned}
$$

After deriving the optimal contract, we will check that (23) is fulfilled in equilibrium, and that (24) is implied by (23).

The following preliminary result is helpful:

Lemma 2 When at the optimum $w_{h}^{U}>0$, then $\Delta w_{h}^{U}=\Delta q$.

Proof. Assume that at the optimum $\Delta w_{h}^{U}<\Delta q$ while $w_{h}^{U}>0$. Let $\epsilon>0$ and $\Delta \epsilon>0$ be such that $w_{h}^{U \prime}=w_{h}^{U}-\epsilon \geq 0$ and $\Delta w_{h}^{U \prime}=\Delta w_{h}^{U}+\Delta \epsilon \leq \Delta q$ with $E U_{g h}=\frac{\left(s_{h}^{U} \Delta w_{h}^{U}\right)^{2}}{2 a_{g}}+s_{h}^{U} w_{h}^{U}=$ $E U_{g h}^{\prime}=\frac{\left(s_{h}^{U} \Delta w_{h}^{U \prime}\right)^{2}}{2 a_{g}}+s_{h}^{U} w_{h}^{U \prime}$. Solving this equation yields $\epsilon=\frac{s_{h}^{U}}{2 a_{g}} \Delta \epsilon\left(2 \Delta w_{h}^{U}+\Delta \epsilon\right)$. By construction, the expected utility of a $g h$ worker is unchanged. However the principal's profit changes: she pays $\Delta \epsilon$ more with probability $e_{g h}^{U \prime}=\frac{\left(\Delta w_{h}^{U}+\Delta \epsilon\right) s_{h}^{U}}{a_{g}}$, but economizes $\epsilon$. Her 
profit thus rises by the amount $\Delta \pi=e_{g h}^{U \prime}\left(\Delta q-\Delta w_{h}^{U \prime}\right)-w_{h}^{U \prime}-\left(e_{g h}^{U}\left(\Delta q-\Delta w_{h}^{U}\right)-w_{h}^{U}\right)$. Substituting for the new value of effort and wages yields: $\Delta \pi=\epsilon+\frac{\Delta \epsilon s_{h}^{U}}{a_{g}}\left(\Delta q-2 \Delta w_{h}^{U}-\Delta \epsilon\right)=$ $\frac{s_{h}^{U} \Delta \epsilon}{a_{g}}\left(\Delta w_{h}^{U}+0.5 \Delta \epsilon\right)+\frac{\Delta \epsilon s_{h}^{U}}{a_{g}}\left(\Delta q-2 \Delta w_{h}^{U}-\Delta \epsilon\right)$. Since by construction, $\Delta w_{h}^{U \prime}=\Delta w_{h}^{U}+\Delta \epsilon \leq \Delta q$, then $\Delta \pi=\frac{s_{h}^{U} \Delta \epsilon}{a_{g}}\left(\Delta q-\Delta w_{h}^{U}-0.5 \Delta \epsilon\right)>0$.

The remainder of the proof proceeds in three steps. In step 1 we show $\epsilon_{h}=0$ allowing us to derive preliminary functional forms for $s_{h}^{U}$ and $w_{h}^{U}$. In step 2 we show $\beta_{g}>0$ implying that the $\left(I R_{g 1}\right)$-constraint is binding. This together with Lemma 2 gives the contract in Proposition 2. In step 3 we show that the firm's profit is positive given this contract and that the solution satisfies (23) and (24) as well as $e_{g 1}^{U}<1, e_{g h}^{U} \leq 1$.

Step 1: To see $\epsilon_{h}=0$ from (22), (23), and (24) we have $s_{h}^{U}=\frac{2 \Delta U_{g}}{B\left(a_{g}+\Delta U_{g}\right)}$. Using (20), (23), (24) and Lemma 2 we get $\epsilon_{h}=0$. Substituting $\epsilon_{h}=0, \beta_{b}=0$ from $(24), s_{h}^{U}=\frac{2 \Delta U_{g}}{B\left(a_{g}+\Delta U_{g}\right)}$ and $\Delta w_{h}^{U}=\Delta q$ in (21) yields $\gamma=\frac{\Delta q^{2}}{a_{g}}+\frac{w_{h}^{U}}{s_{h}^{U}}>0$. This implies in (17) that $s_{h}^{U}=\frac{a_{g}+\Delta U_{g}}{\Delta U_{g}}$ and $w_{h}^{U}=\frac{a_{g}}{\left(s_{h}^{U}-1\right) s_{h}^{U}}-s_{h}^{U} \frac{\Delta q^{2}}{2 a_{g}}+\frac{U_{g}}{s_{h}^{U}}$.

Step 2: Next we show $\beta_{g}>0$. First, we show $\beta_{g} \frac{\Delta U_{g}}{a_{g}}+\alpha>0$. Substituting for $B$ and $D$ in (20) and rearranging yields: $\beta_{g} \frac{\Delta U_{g}}{a_{g}}+\alpha=\frac{2}{\left(s_{h}^{U}\right)^{2}}\left(1-\frac{a_{g}}{a_{g}+\Delta U_{g}}\left(\frac{\Delta q s_{h}^{U}}{\Delta U_{g}}-2-2 \frac{\left(s_{h}^{U}-1\right)}{a_{g}} \underline{U}_{g}\right)\right)$. After some computations and given Assumption A.2 it can be shown that $\beta_{g} \frac{\Delta U_{g}}{a_{g}}+\alpha>0$. This implies $\beta_{g}>0$ and $\alpha=0$. Assume, to the contrary, $\alpha>0$. From (14) we have $E U_{g h}=\underline{U}_{g}$ and thus $\Delta U_{g}=0$. Then, the individual rationality constraint $\left(I R_{g 1}\right)$ with $\frac{\Delta U_{g}^{2}}{2 a_{g}} \geq 2 \underline{U}_{g}$ cannot be fulfilled. Given that $\alpha=0$, we must have $\beta_{g}>0$. Hence, $\frac{\Delta U_{g}^{2}}{2 a_{g}}=2 \underline{U}_{g}$ or $\Delta U_{g}=2 \sqrt{a_{g} \underline{U}_{g}}$. We therefore have $s_{h}^{U}=1+\frac{1}{2} \sqrt{\frac{a_{g}}{\underline{U}_{g}}}>1$. Substituting this value in $w_{h}^{U}$ from step 1 together with Lemma 2 yields the three conditions of Proposition 2.

Step 3: It needs to be shown that the firm's profit is positive given these specifications. We have $\pi^{U}=\frac{2 e_{g 1}^{U}}{1+e_{g 1}^{U}}\left(\Delta q-w_{h}^{U}\right)+2 q$. Substituting for $s_{h}^{U}=\frac{e_{g 1}^{U}}{1+e_{g 1}^{U}}$, profit is positive if $\Delta q\left(1+e_{g 1}^{U}\right)-\left(e_{g 1}^{U}\right)^{2} a_{g}+\frac{\left(2 e_{g 1}^{U}+1\right)}{e_{g 1}^{U}} \frac{\Delta q^{2}}{2 a_{g}}+\frac{1+2 e_{g 1}^{U}}{e_{g 1}^{U}} q>0$. A sufficient condition is $\frac{\Delta q}{q}\left(1+e_{g 1}^{U}\right)-$ $\left(e_{g 1}^{U}\right)^{2} \frac{a_{g}}{q}+\frac{1+2 e_{g 1}^{U}}{e_{g 1}^{U}}>0$. Let $a=\frac{a_{g}}{q}$ and $\Delta=\frac{\Delta q}{q}$ implying $e_{g 1}^{U}=\frac{\sqrt{2}}{a} \sqrt{2 a+\Delta^{2}}$. Then, A.2 becomes $a \geq 37.5$ and $\Delta \leq 5$. Thus, profit is positive if $u(a, \Delta)=\Delta\left(1+e_{g 1}^{U}(a, \Delta)\right)-$ $\left(e_{g 1}^{U}\right)^{2}(a, \Delta) a+\frac{1+2 e_{g 1}^{U}(a, \Delta)}{e_{g 1}^{U}(a, \Delta)}>0$. As both $\frac{\partial u(a, \Delta)}{\partial a}, \frac{\partial u(a, \Delta)}{\partial \Delta}>0$, it suffices to show that $u(a, \Delta)$ is positive for the lowest values $a$ and $\Delta$. We have $u(37.5,0) \sim 1.06$.

Finally, we need to check that the solution satisfies (23), (24) and $e_{g 1}^{U}<1, e_{g h}^{U} \leq 1$. For (23) we obtain as a sufficient condition $2 \sqrt{\sqrt{\left(q a_{g}+\frac{\Delta q^{2}}{2}\right)}}>\frac{\Delta q}{\sqrt{a_{g}}}+\frac{\Delta q}{2} \sqrt{\frac{1}{q}}$. The LHS is increasing in $a_{g}$ and the RHS decreasing. Given A.2, we can thus set $a_{g}=37.5 q$. For 
$\frac{\Delta q}{q}<5$ (by A.2), the sufficient condition is fulfilled. Second, we check $e_{g 1}^{U}<1$. This is equivalent to $a_{g}>2 q\left(1+\sqrt{1+\frac{\Delta q^{2}}{2 q^{2}}}\right)$. According to A.2, $a_{g} \geq 37.5 q$. Thus we have to show $37.5 q>2 q\left(1+\sqrt{1+\frac{\Delta q^{2}}{2 q^{2}}}\right)$. This is equivalent to $\Delta q<\sqrt{2\left(\left(\frac{37.5}{2}-1\right)^{2}-1\right)} q \approx 25 q$ and fulfilled given A.2. Third, we turn to (24). $E U_{b 1}<2 \underline{U}_{b}$ is equivalent to $\left(s_{h}^{U}\right)^{2}\left(\frac{1}{a_{g}}-\frac{1}{a_{b}}\right)>$ $\left(\frac{1}{a_{g}}-\frac{1}{a_{b}}\right)-\frac{4}{\Delta q^{2}}\left(\sqrt{a_{b} q+\frac{\Delta q^{2}}{2}}-\sqrt{a_{g} q+\frac{\Delta q^{2}}{2}}\right)$. The second term on the RHS is negative since $a_{b}>a_{g}$. Thus, a sufficient condition is $\left(s_{h}^{U}\right)^{2}\left(\frac{1}{a_{g}}-\frac{1}{a_{b}}\right)>\left(\frac{1}{a_{g}}-\frac{1}{a_{b}}\right)$, which is always fulfilled since $s_{h}^{U}>2$. Thus, a low-ability worker would never like to enter the firm. We finish with $e_{g h}^{U} \leq 1$ which is equivalent to $a_{g}^{2}-4 a_{g} \Delta q+2 \Delta q^{2}+a_{g}^{2} \frac{2 a_{g} q}{\Delta q^{2}+2 a_{g} q} \geq 0$. A sufficient condition for this to hold is $a_{g} \geq(2+\sqrt{2}) \Delta q$. Given assumption A.2 we have $a_{g} \geq 37.5 q$ and thus have to show $37.5 q \geq(2+\sqrt{2}) \Delta q$. This is fulfilled since $\frac{\Delta q}{q}<5$ by A.2. QED

\section{Proof of Proposition 3}

The proof is similar to the one of Proposition 3 in Auriol and Renault (2001). However, we here consider two types of agents, and identify a separating equilibrium with shutdown of the less productive type $\left(I R_{b 1}\right)$. By offering optimal incentive contracts the firm is able to sort out at zero cost the most productive workers. As in the Proof of Proposition 2, we first show that it is optimal for juniors to receive minimal wages and status: $\Delta w_{1}^{S}=w_{1}^{S}=s_{1}^{S}=0$. We avoid introducing additional notation by neglecting here the index for possible different types of workers. The reasoning holds true for any type of worker.

If $s_{1}^{S}=0$, then it is optimal to set $w_{1}^{S}=\Delta w_{1}^{S}=0$. Thus the proof of the result amounts to showing that any optimal contract is such that $s_{1}^{S}=0$. Suppose to the contrary that $s_{1}^{S}>0$. At some date $t$ the principal may switch to the new contract where the first term is status, the second fixed wage, and the third bonus: $c_{1}^{S^{\prime}}=(0,0,0)$;

$c_{h}^{S^{\prime}}=\left(s_{h}^{S}+s_{1}^{S}, \frac{s_{h}^{S} w_{h}^{S}+s_{1}^{S}\left(w_{1}^{S}+\Delta w_{1}^{S}\right)}{s_{h}^{S}+s_{1}^{S}}, \frac{s_{h}^{S} \Delta w_{h}^{S}}{s_{h}^{S}+s_{1}^{S}}\right) ; c_{l}^{S^{\prime}}=\left(s_{l}^{S}+s_{1}^{S}, \frac{s_{l}^{S} w_{l}^{S}+s_{1}^{S} w_{1}^{S}}{s_{l}^{S}+s_{1}^{S}}, \frac{s_{l}^{S} \Delta w_{l}^{S}}{s_{l}^{S}+s_{1}^{S}}\right)$.

If each generation from $t$ on is offered these contracts, by construction whatever the worker's type the young's expected intertemporal utility is held constant. Basically, the young's wages are transferred from the first to the second period while being divided by the ratio of the original period 1 status to the new second period status $\frac{s_{1}^{S}}{s_{1}^{S}+s_{p}^{S}}, p \in\{l, h\}$, so that the increase in status exactly compensates for the decrease in income. On the other hand, the utility of an old agent is increased (by $s_{1}^{S} w_{1}^{S}$ for the $l$ type and $s_{1}^{S}\left(w_{1}^{S}+\Delta w_{1}^{S}\right)$ for the $h$ type). Furthermore, as the overall incentives do not change, all effort levels are maintained. Let $E w_{1}^{S}=w_{1}^{S}+\mu\left(e_{1}^{S}\right) \Delta w_{1}^{S}, E w_{h}^{S}=w_{h}^{S}+\mu\left(e_{h}^{S}\right) \Delta w_{h}^{S}$ and $E w_{l}^{S}=w_{l}^{S}+\mu\left(e_{l}^{S}\right) \Delta w_{l}^{S}$ be the expected wage bill for junior and senior workers in the original contract and $E w_{1}^{S^{\prime}}=0$, $E w_{h}^{S^{\prime}}=\frac{s_{h}^{S}}{s_{h}^{S}+s_{1}^{S}} E w_{h}^{S}+\frac{s_{1}^{S}}{s_{h}^{S}+s_{1}^{S}}\left(w_{1}^{S}+\Delta w_{1}^{S}\right)$, and $E w_{l}^{S^{\prime}}=\frac{s_{l}^{S}}{s_{l}^{S}+s_{1}^{S}} E w_{l}^{S}+\frac{s_{1}^{S}}{s_{l}^{S}+s_{1}^{S}} w_{1}^{S}$ in the new contract. 
It is straightforward to check that the intertemporal wage bill for each generation is lower. Hence, a steady state with $s_{1}^{S}>0$ cannot be part of any optimal solution.

Let $\Delta U_{i}=E U_{i h}-E U_{i l}$. The principal is maximizing expected profits:

$$
\max E \Pi=e_{g 1}^{S}\left(\Delta q+e_{g h}^{S}\left(\Delta q-\Delta w_{h}^{S}\right)-w_{h}^{S}\right)+\left(1-e_{g 1}^{S}\right)\left(e_{g h}^{S}\left(\Delta q-\Delta w_{l}^{S}\right)-w_{l}^{S}\right)+2 q
$$

subject to $E U_{g l}=\frac{\left(s_{l}^{S} \Delta w_{l}^{S}\right)^{2}}{2 a_{g}}+s_{l}^{S} w_{l}^{S} \geq \underline{U}_{g}\left(I R_{g l}\right), E U_{g 1}=\frac{\Delta U_{g}^{2}}{2 a_{g}}+E U_{g l} \geq 2 \underline{U}_{g}\left(I R_{g 1}\right), E U_{b 1}=$ $\frac{\Delta U_{b}^{2}}{2 a_{b}}+E U_{b l}<2 \underline{U}_{b}\left(I R_{b 1}\right), 2=e_{g 1}^{S} s_{h}^{S}+\left(1-e_{g 1}^{S}\right) s_{l}^{S}$ (status feasibility), $w_{h}^{S} \geq 0 ; w_{l}^{S} \geq 0$ $(L L), e_{g 1}^{S}=\frac{\Delta U_{g}}{a_{g}}\left(I C_{g 1}\right), e_{g p}^{S}=\frac{\Delta w_{p}^{S} s_{p}^{S}}{a_{g}}$ for $p=l, h\left(I C_{g p}\right)$, and $s_{h}^{S} \Delta w_{h}^{S} \leq a_{g} ; s_{l}^{S} \Delta w_{l}^{S} \leq a_{g}$. Substituting the effort functions from $\left(I C_{g 1}\right)$ and $\left(I C_{g p}\right)$, the Lagrangian is:

$$
\begin{aligned}
L & =\frac{\Delta U_{g}}{a_{g}}\left(\Delta q+\frac{s_{h}^{S} \Delta w_{h}^{S}}{a_{g}}\left(\Delta q-\Delta w_{h}^{S}\right)-w_{h}^{S}\right)+\left(1-\frac{\Delta U_{g}}{a_{g}}\right)\left(\frac{s_{l}^{S} \Delta w_{l}^{S}}{a_{g}}\left(\Delta q-\Delta w_{l}^{S}\right)-w_{l}^{S}\right)+2 q \\
& +\alpha\left(\frac{\left(s_{l}^{S} \Delta w_{l}^{S}\right)^{2}}{2 a_{g}}+s_{l}^{S} w_{l}^{S}-\underline{U}_{g}\right)+\beta_{g}\left(\frac{\Delta U_{g}^{2}}{2 a_{g}}+E U_{g l}-2 \underline{U}_{g}\right)+\beta_{b}\left(2 \underline{U}_{b}-\frac{\Delta U_{b}^{2}}{2 a_{b}}-E U_{b l}\right) \\
& +\gamma\left(2-\frac{\Delta U_{g}}{a_{g}} s_{h}^{S}-\left(1-\frac{\Delta U_{g}}{a_{g}}\right) s_{l}^{S}\right)+\lambda_{l} w_{l}^{S}+\lambda_{h} w_{h}^{S}+\epsilon_{h}\left(a_{g}-\Delta w_{h}^{S} s_{h}^{S}\right)+\epsilon_{l}\left(a_{g}-\Delta w_{l}^{S} s_{l}^{S}\right)
\end{aligned}
$$

Define $G:=\Delta q+\frac{s_{h}^{S} \Delta w_{h}^{S}}{a_{g}}\left(\Delta q-\Delta w_{h}^{S}\right)-w_{h}^{S}-\frac{s_{l}^{S} \Delta w_{l}^{S}}{a_{g}}\left(\Delta q-\Delta w_{l}^{S}\right)+w_{l}^{S}+\beta_{g} \Delta U_{g}-\gamma\left(s_{h}^{S}-s_{l}^{S}\right)$. From the Lagrangian, we derive the following conditions:

$$
\begin{aligned}
\alpha \geq 0 ; \beta_{g} \geq 0 ; \beta_{b} \geq 0 ; \gamma \geq 0 ; \lambda_{l} \geq 0 ; \lambda_{h} \geq 0 ; \epsilon_{l} \geq 0 ; \epsilon_{h} \geq 0 & \\
\alpha\left(E U_{g l}-\underline{U}_{g}\right) & =0 \\
\beta_{g}\left(\frac{\Delta U_{g}^{2}}{2 a_{g}}+E U_{g l}-2 \underline{U}_{g}\right) & =0 \\
\beta_{b}\left(2 \underline{U}_{b}-\frac{\Delta U_{b}^{2}}{2 a_{b}}-E U_{b l}\right) & =0 \\
\gamma\left(2-\frac{\Delta U_{g}}{a_{g}} s_{h}^{S}-\left(1-\frac{\Delta U_{g}}{a_{g}}\right) s_{l}^{S}\right) & =0 \\
\epsilon_{h}\left(a_{g}-\Delta w_{h}^{S} s_{h}^{S}\right)=0 ; \epsilon_{l}\left(a_{g}-\Delta w_{l}^{S} s_{l}^{S}\right) & =0 \\
\frac{\partial L}{\partial \Delta w_{h}^{S}}=\frac{s_{h}^{S} \Delta w_{h}^{S}}{a_{g}^{2}} G-\frac{s_{h}^{S} \Delta w_{h}^{S}}{a_{b}^{2}} \beta_{b} \Delta U_{b}+\frac{\Delta U_{g}}{a_{g}^{2}}\left(\Delta q-2 \Delta w_{h}^{S}\right) & =\epsilon_{h}=0
\end{aligned}
$$




$$
\begin{aligned}
& \frac{\partial L}{\partial s_{h}^{S}}=\left(\frac{s_{h}^{S} \Delta w_{h}^{S}}{a_{g}^{2}}+\frac{w_{h}^{S}}{a_{g} \Delta w_{h}^{S}}\right) G-\left(\frac{s_{h}^{S} \Delta w_{h}^{S}}{a_{b}^{2}}+\frac{w_{h}^{S}}{a_{b} \Delta w_{h}^{S}}\right) \beta_{b} \Delta U_{b} \\
& +\frac{\Delta U_{g}}{a_{g}^{2}}\left(\Delta q-\Delta w_{h}^{S}\right)-\gamma\left(\frac{\Delta U_{g}}{a_{g} \Delta w_{h}^{S}}\right)-\epsilon_{h}=0 \\
& \frac{\partial L}{\partial \Delta w_{l}^{S}}=\frac{s_{l}^{S} \Delta w_{l}^{S}}{a_{g}}\left(-\frac{G}{a_{g}}+\beta_{g}+\alpha\right)-\frac{s_{l}^{S} \Delta w_{l}^{S}}{a_{b}} \beta_{b}\left(1-\frac{\Delta U_{b}}{a_{b}}\right)+ \\
& \left(1-\frac{\Delta U_{g}}{a_{g}}\right)\left(\frac{\Delta q-2 \Delta w_{l}^{S}}{a_{g}}\right)-\epsilon_{l}=0 \\
& \frac{\partial L}{\partial s_{l}^{S}}=\left(\frac{s_{l}^{S} \Delta w_{l}^{S}}{a_{g}}+\frac{w_{l}^{S}}{\Delta w_{l}^{S}}\right)\left(-\frac{G}{a_{g}}+\beta_{g}+\alpha\right)-\left(\frac{s_{l}^{S} \Delta w_{l}^{S}}{a_{b}}+\frac{w_{l}^{S}}{\Delta w_{l}^{S}}\right) \beta_{b}\left(1-\frac{\Delta U_{b}}{a_{b}}\right) \\
& +\left(1-\frac{\Delta U_{g}}{a_{g}}\right) \frac{\Delta q-\Delta w_{l}^{S}}{a_{g}}-\frac{\gamma}{\Delta w_{l}^{S}}\left(1-\frac{\Delta U_{g}}{a_{g}}\right)-\epsilon_{l}=0 \\
& \frac{\partial L}{\partial w_{h}^{S}}=s_{h}^{S}\left(\frac{G}{a_{g}}-\beta_{b} \frac{\Delta U_{b}}{a_{b}}\right)-\frac{\Delta U_{g}}{a_{g}}+\lambda_{h}=0 \\
& \frac{\partial L}{\partial w_{l}^{S}}=s_{l}^{S}\left(-\frac{G}{a_{g}}+\beta_{b} \frac{\Delta U_{b}}{a_{b}}+\beta_{g}-\beta_{b}+\alpha\right)-\left(1-\frac{\Delta U_{g}}{a_{g}}\right)+\lambda_{l}=0
\end{aligned}
$$

Depending on parameters different solutions can occur. We focus on the case where in equilibrium:

$$
\begin{aligned}
& w_{h}^{S}>0 \text { and } w_{l}^{S}>0 \Rightarrow \lambda_{h}=\lambda_{l}=0 \\
& \beta_{b}=0 \Rightarrow E U_{b 1}<2 \underline{U}_{b} \\
& E U_{g l}=\underline{U}_{g} \Rightarrow \alpha>0 \\
& \beta_{g}>0, \text { which with } \alpha>0, \text { implies that } \Delta U_{g}=\sqrt{2 a_{g} \underline{U}_{g}} \text { and } e_{g 1}^{S}=\sqrt{\frac{2 \underline{U}_{g}}{a_{g}}}
\end{aligned}
$$

Note that with (40) we focus on the case where unsuccessful seniors receive only their reservation utility, i.e., where the principal is setting incentives for juniors to work. It will be checked that all these conditions are fulfilled at equilibrium and that condition (39) is implied by (38).

The remainder of the proof proceeds in two steps. In step 1 we show $\epsilon_{h}=\epsilon_{l}=0$. This together with conditions (38) to (41) gives the contract in Proposition 3. In step 2 we show that the firm's profit is positive given this contract and that the solution satisfies conditions (38) to (41) as well as $e_{g 1}^{S}<1 ; e_{g l}^{S}, e_{g h}^{S} \leq 1$. 
Step 1: Lemma 2 derived in the Proof of Proposition 2 applies for $w_{h}$ and $w_{l}$. From (36), (38) and (39) we have $s_{h}^{S}=\frac{\Delta U_{g}}{G}$. From (32) and (39) we get $\frac{\Delta U_{g}}{a_{g}^{2}}\left(\Delta q-\Delta w_{h}^{S}\right)=\epsilon_{h}$. Similarly, from (34) and (37) we get: $\left(1-\frac{\Delta U_{g}}{a_{g}}\right) \frac{1}{a_{g}}\left(\Delta q-\Delta w_{l}^{S}\right)=\epsilon_{l}$. Lemma 2 with (38) then implies $\epsilon_{h}=\epsilon_{l}=0$. Substituting (32) in (33) and applying (38) and (39) to (36), we get $\gamma=\frac{w_{h}^{S}}{s_{h}^{S}}+\frac{\Delta q^{2}}{a_{g}}$.

From (36), (37) and (38) it follows that $\beta_{g}+\alpha=\frac{1}{s_{l}^{S}}\left(1-\frac{\Delta U_{g}}{a_{g}}\right)+\frac{\Delta U_{g}}{a_{g} s_{h}^{S}}$. Since $\frac{\Delta U_{g}}{a_{g}}=e_{g 1}^{S}<1$ we have $\beta_{g}+\alpha>0$. Substituting $\beta_{g}+\alpha$ in (34) and (35), we get $\gamma=\frac{w_{l}^{S}}{s_{l}^{S}}+\frac{\Delta q^{2}}{a_{g}}$. Equating these two expressions for $\gamma$ it follows that $\left(s_{h}^{S}, s_{l}^{S}\right)$ is such that: $\frac{w_{h}^{S}}{s_{h}^{S}}=\frac{w_{l}^{S}}{s_{l}^{S}}$. Moreover we have $\gamma>0$ so that $\frac{\Delta U_{g}}{a_{g}} s_{h}^{S}+\left(1-\frac{\Delta U_{g}}{a_{g}}\right) s_{l}^{S}=2$. Given these specifications as well as (40) and (41) and using $\frac{w_{h}^{S}}{s_{h}^{S}}=\frac{w_{l}^{S}}{s_{l}^{S}}$ and $\frac{\left(s_{l}^{S} \Delta q\right)^{2}}{2 a_{g}}+s_{l}^{S} w_{l}^{S}=\underline{U}_{g}$ we get the solution in Proposition 3 .

Step 2: It needs to be shown that the firm's profit $\pi^{S}$ is positive given these specifications. We have $\pi^{S}=e_{g 1}^{S}\left(\Delta q-w_{h}^{S}\right)-\left(1-e_{g 1}^{S}\right) w_{l}^{S}+2 q$. Using the fact that $\frac{w_{h}^{S}}{s_{h}^{S}}=\frac{w_{l}^{S}}{s_{l}^{S}}$, this is equivalent to: $\pi^{S}=2 q+e_{g 1}^{S} \Delta q-w_{l}^{S}\left(1+e_{g 1}^{S} \frac{s_{h}^{S}-s_{l}^{S}}{s_{l}^{S}}\right)$. Since $e_{g 1}^{S}=\sqrt{\frac{2 \underline{U}_{g}}{a_{g}}}$ and since $s_{h}^{S}=$ $s_{l}^{S} \sqrt{\sqrt{\frac{2 a_{g}}{\underline{G}_{g}}}+1}$ we deduce that $\pi^{S}=2 q+e_{g 1}^{S} \Delta q-w_{l}^{S}\left\{1+\sqrt{\frac{2 \underline{\underline{U}}_{g}}{a_{g}}}\left(\sqrt{\sqrt{\frac{2 a_{g}}{\underline{U}_{g}}}+1}-1\right)\right\}=2 q+$ $e_{g 1}^{S} \Delta q-\frac{2 w_{l}^{S}}{s_{l}^{S}}$. Substituting $w_{l}^{S}=\frac{\underline{U}_{g}}{s_{l}^{S}}-\frac{s_{l}^{S} \Delta q^{2}}{2 a_{g}}=\frac{q}{s_{l}^{S}}+\frac{\Delta q^{2}}{2 a_{g} s_{l}^{S}}-\frac{s_{l}^{S} \Delta q^{2}}{2 a_{g}}$ yields $\pi^{S}=2 q+e_{g 1}^{S} \Delta q-\frac{2 w_{l}^{S}}{s_{l}^{S}}=$ $e_{g 1}^{S} \Delta q+\left(2 q+\frac{\Delta q^{2}}{a_{g}}\right)\left(1-\frac{1}{\left(s_{l}^{S}\right)^{2}}\right)>0$ since $s_{l}^{S}>1$.

Finally, we need to check that the solution satisfies conditions (38) to (41), and $e_{g 1}^{U}<$ $1, e_{g l}^{S}, e_{g h}^{S} \leq 1$. For $(38)$ we show $w_{l}^{S}>0$ which implies $w_{h}^{S}=w_{l}^{S} \sqrt{\sqrt{\frac{2 a_{g}}{\underline{U}_{g}}}+1}>0$. We need to show $\frac{\underline{U}_{g}}{s_{l}^{S}}>\frac{s_{l}^{S} \Delta q^{2}}{2 a_{g}}$. A sufficient condition is $a_{g} \geq \frac{3}{2} \frac{\Delta q^{2}}{q}$. Given assumption A.2 we have $a_{g} \geq 37.5 q$. We thus have to show $37.5 \geq \frac{3}{2} \frac{\Delta q^{2}}{q^{2}}$. This is fulfilled for $\frac{\Delta q}{q} \leq 5$ as given by assumption A.2. Second, condition (39) is satisfied if $E U_{b 1}=$ $\frac{1}{2 a_{b}}\left(\frac{\Delta q^{2}}{2 a_{b}}\left(\left(s_{h}^{S}\right)^{2}-\left(s_{l}^{S}\right)^{2}\right)+s_{h}^{S} w_{h}^{S}-s_{l}^{S} w_{l}^{S}\right)^{2}+\frac{\left(s_{l}^{S} \Delta q\right)^{2}}{2 a_{b}}+s_{l}^{S} w_{l}^{S}<2 \underline{U}_{b}$. The condition simplifies to $\frac{\Delta q^{2}\left(\frac{\left(s_{l}^{S}\right)^{2}}{2}-1\right)+\frac{a_{g}}{\underline{U}_{g}}\left(q-\frac{\Delta q^{2}}{2 a_{g}}\left(\left(s_{l}^{S}\right)^{2}-1\right)+\frac{\left(s_{l}^{S}\right)^{2} \Delta q^{2}}{2} \frac{1}{a_{b}}\right)^{2}}{q+\frac{\Delta q^{2}}{2 a_{g}}\left(\left(s_{l}^{S}\right)^{2}-1\right)}<a_{b}$. Since the $L H S<a_{g}$ and by assumption $a_{g}<a_{b}$ this condition is always fulfilled.

Next we check (41) by checking that $\beta_{g}>0$. Since $\Delta w_{h}^{S}=\Delta w_{l}^{S}=\Delta q$ we have $G=$ $\Delta q-\left(w_{h}^{S}-w_{l}^{S}\right)+\beta_{g} \Delta U_{g}-\gamma\left(s_{h}^{S}-s_{l}^{S}\right)$. Substituting $\gamma=\frac{w_{h}^{S}}{s_{h}^{S}}+\frac{\Delta q^{2}}{a_{g}}=\left(\frac{w_{l}^{S}}{s_{l}^{S}}+\frac{\Delta q^{2}}{a_{g}}\right)$ yields $G=\Delta q-2\left(w_{h}^{S}-w_{l}^{S}\right)-\frac{\Delta q^{2}}{a_{g}}\left(s_{h}^{S}-s_{l}^{S}\right)+\beta_{g} \Delta U_{g}$. Let $A=\sqrt{\sqrt{\frac{2 a_{g}}{\underline{U}_{g}}}+1}-1$. We have $w_{h}^{S}=(A+1) w_{l}^{S}=(A+1)\left(\frac{\underline{U}_{g}}{s_{l}^{S}}-\frac{s_{l}^{S} \Delta q^{2}}{2 a_{g}}\right)$ and $s_{h}^{S}=(A+1) s_{l}^{S}$. Substituting these values 
in $G$ yields $G=\Delta q-\frac{2 A \underline{U}_{g}}{s_{l}^{S}}+\beta_{g} \Delta U_{g}$. Moreover since $\beta_{b}=\lambda_{h}=0$ we deduce from (36) that $G=\frac{\Delta U_{g}}{s_{h}^{S}}$, where $\Delta U_{g}=\sqrt{2 a_{g} \underline{U}_{g}}$. Combining both equations of $G$ yields $(i) \beta_{g}=\frac{1}{s_{h}^{S}}+$ $\sqrt{\frac{2 \underline{U}_{g}}{a_{g}}} \frac{A}{s_{l}^{S}}-\frac{\Delta q}{\sqrt{2 a_{g} \underline{U}_{g}}}$. We deduce that $\beta_{g}>0$ if and only if $s_{l}^{S} \Delta q<\frac{\sqrt{2 a_{g} \underline{U}_{g}}}{A+1}+2 \underline{U}_{g} A$. Substituting $A$ by its value, this inequality is equivalent to $(i i) 2 \underline{U}_{g}+s_{l}^{S} \Delta q<\frac{3 \sqrt{2 a_{g} \underline{U}_{g}}+2 \underline{U}_{g}}{\sqrt{\sqrt{\frac{2 a_{g}}{\underline{\underline{G}}_{g}}}+1}}$. Dividing right and left by $\underline{U}_{g}$ and substituting $\underline{U}_{g}$ by its value in $\sqrt{\frac{2 a_{g}}{\underline{U}_{g}}}=\frac{2 a}{\sqrt{2 a+\Delta^{2}}}$, equation $(i i)$ becomes $\left(1+\frac{s_{l}^{S} a}{2 a+\Delta^{2}} \Delta\right)^{2}\left(1+\frac{2 a}{\sqrt{2 a+\Delta^{2}}}\right)<\left(1+\frac{3 a}{\sqrt{2 a+\Delta^{2}}}\right)^{2}$, with $s_{l}^{S}=\frac{2}{1+\frac{\sqrt{2 a+\Delta^{2}}}{a}\left(\sqrt{\frac{2 a}{\sqrt{2 a+\Delta^{2}}}+1}-1\right)}$. On can check that this condition holds for all values of $a$ and $\Delta$ satisfying A.2. Thus, $\beta_{g}>0$.

We then check condition (40) by checking that $\alpha>0$. We have $\beta_{g}+\alpha=\frac{1}{s_{l}^{S}}-\frac{\Delta U_{g}}{a_{g}} \frac{s_{h}^{S}-s_{l}^{S}}{s_{h}^{S} S_{l}^{S}}$ so that $\alpha=\frac{1}{s_{l}^{S}}-\sqrt{\frac{2 \underline{U}_{g}}{a_{g}}} \frac{s_{h}^{S}-s_{l}^{S}}{s_{h}^{S} s_{l}^{S}}-\beta_{g}$. Substituting $\beta_{g}$ by its value from $(i)$ above yields $\alpha=\left(1-\sqrt{\frac{2 \underline{U}_{g}}{a_{g}}}\right) \frac{s_{h}^{S}-s_{l}^{S}}{s_{h}^{S} s_{l}^{S}}-\sqrt{\frac{2 \underline{U}_{g}}{a_{g}}} \frac{A}{s_{l}^{S}}+\frac{\Delta q}{\sqrt{2 a_{g} \underline{U}_{g}}}=\frac{A}{(A+1) s_{l}^{S}}\left(1-\sqrt{\frac{2 \underline{U}_{g}}{a_{g}}}(A+2)\right)+\frac{\Delta q}{\sqrt{2 a_{g} \underline{U}_{g}}}$. Thus, $\alpha>0$ if $\sqrt{\frac{a_{g}}{2 \underline{U}_{g}}}+\frac{A+1}{A} \frac{s_{l}^{S} \Delta q}{2 \underline{U}_{g}}>A+2$. Inserting values for $A, \underline{U}_{g}$, and $s_{l}^{S}$ gives $\frac{a}{\sqrt{2 a+\Delta^{2}}}+$ $\frac{\sqrt{\frac{2 a}{\sqrt{2 a+\Delta^{2}}}+1}}{\sqrt{\frac{2 a}{\sqrt{2 a+\Delta^{2}}}+1}-1} \frac{2 a}{1+\frac{\sqrt{2 a+\Delta^{2}}}{a}\left(\sqrt{\frac{2 a}{\sqrt{2 a+\Delta^{2}}}+1}-1\right)} \frac{\Delta}{2 a+\Delta^{2}}>\sqrt{\frac{2 a}{\sqrt{2 a+\Delta^{2}}}+1}+1$. One can check that this inequality holds for all values of $a$ and $\Delta$ satisfying A.2. Thus, $\alpha>0$.

Next, we show $e_{g 1}^{S}<1$. This is fulfilled since $\sqrt{2} e_{g 1}^{S}=e_{g 1}^{U}<1$. We finish with $e_{g h}^{S}=$ $\frac{s_{h}^{S} \Delta q}{a_{g}}<1$ which implies $e_{g l}^{S}=\frac{s_{l}^{S} \Delta q}{a_{g}}<1$. We have to have $a_{g}>s_{h}^{S} \Delta q$ which is equivalent to $a_{g}-\sqrt{2 a_{g} \underline{U}_{g}}+\sqrt{2 a_{g} \underline{U}_{g}} \sqrt{\sqrt{\frac{2 a_{g}}{\underline{U}_{g}}}+1}>2 \Delta q \sqrt{\sqrt{\frac{2 a_{g}}{\underline{U}_{g}}}+1}$. Given $e_{g 1}^{S}<1$ we have $a_{g}>\sqrt{2 a_{g} \underline{U}_{g}}$. A sufficient condition is thus $\sqrt{2 a_{g} \underline{U}_{g}} \geq 2 \Delta q$ which is true as shown above for condition (38). QED

\section{Proof of Proposition 4} if:

Profit with up-or-out is higher than profit with standard promotion practices if and only

$$
\frac{2 e_{g 1}^{U}}{1+e_{g 1}^{U}}\left(\Delta q-w_{h}^{U}\right)+2 q>e_{g 1}^{S}\left(\Delta q-w_{h}^{S}\right)-\left(1-e_{g 1}^{S}\right) w_{l}^{S}+2 q
$$


Defining $\Delta \Pi$ as the difference in profits between up-or-out and standard promotion practices and using that $e_{g 1}^{S}=\frac{1}{\sqrt{2}} e_{g 1}^{U}$, we find

$$
\Delta \Pi=\frac{2 e_{g 1}^{U}}{1+e_{g 1}^{U}}\left(\Delta q-w_{h}^{U}\right)-\frac{e_{g 1}^{U}}{\sqrt{2}}\left(\Delta q-w_{h}^{S}\right)+\left(1-\frac{e_{g 1}^{U}}{\sqrt{2}}\right) w_{l}^{S} .
$$

Using from Proposition $2 e_{g 1}^{U}=\sqrt{2} \sqrt{\frac{2 \underline{U_{g}}}{a_{g}}}$ and from Proposition $3 w_{h}^{S}=w_{l}^{S} \sqrt{\frac{2 \sqrt{2}}{e_{g 1}^{U}}+1}$ and $s_{l}^{S}=\frac{2}{1+\frac{e_{g 1}^{U}}{\sqrt{2}}\left(\sqrt{\frac{2 \sqrt{2}}{e_{g 1}^{U}}+1}-1\right)}$ we obtain:

$$
\Delta \Pi=\left(\frac{2 e_{g 1}^{U}}{1+e_{g 1}^{U}}-\frac{e_{g 1}^{U}}{\sqrt{2}}\right) \Delta q-\frac{2 e_{g 1}^{U}}{1+e_{g 1}^{U}} w_{h}^{U}+2 \frac{w_{l}^{S}}{s_{l}^{S}} .
$$

Furthermore, we have from Proposition $2 w_{h}^{U}=\frac{\left(e_{g 1}^{U}\right)^{2}}{1+e_{g 1}^{U}} a_{g}-\frac{\left(2 e_{g 1}^{U}+1\right)}{e_{g 1}^{U}\left(1+e_{g 1}^{U}\right)} \frac{\Delta q^{2}}{2 a_{g}}+\frac{e_{g 1}^{U}}{1+e_{g 1}^{U}} q$ and from Proposition $3 w_{l}^{S}=\frac{\left(e_{g 1}^{U}\right)^{2}}{4 s_{l}^{S}} a_{g}-\frac{s_{l}^{S} \Delta q^{2}}{2 a_{g}}$. Hence,

$$
\Delta \Pi=\left(\frac{2 e_{g 1}^{U}}{1+e_{g 1}^{U}}-\frac{e_{g 1}^{U}}{\sqrt{2}}\right) \Delta q+\frac{e_{g 1}^{U 2}}{\left(1+e_{g 1}^{U}\right)^{2}} \frac{a_{g}}{8}\left[\left(1+\frac{e_{g 1}^{U}}{\sqrt{2}}\left(\sqrt{\frac{2 \sqrt{2}}{e_{g 1}^{U}}+1}-1\right)\right)^{2}\left(1+e_{g 1}^{U}\right)^{2}-16\left(e_{g 1}^{U}+\frac{\underline{U}_{g}}{a_{g}}\right)\right]
$$

In a way similar to the proof of Proposition 2, we re-scale the problem in function of $q$ with $a=\frac{a_{g}}{q}$ and $\Delta=\frac{\Delta q}{q}$. Given A.2 we have $a \geq 37.5$ and $\Delta \leq 5$. Then,

$$
e_{g 1}^{U}=\frac{\sqrt{2}}{a} \sqrt{2 a+\Delta^{2}} \in\left(0, \frac{10 \sqrt{2}}{37.5}\right] .
$$

It is straightforward to check that $e_{g 1}^{U}$ is decreasing and convex in $a$, and that it is increasing and convex in $\Delta$. It hence reaches its maximum for $a=37.5$ and $\Delta=5$. Equation (44) is equivalent to

$$
\frac{\Delta \Pi(a, \Delta)}{q}=\left(\frac{2 e_{g 1}^{U}}{1+e_{g 1}^{U}}-\frac{e_{g 1}^{U}}{\sqrt{2}}\right) \Delta+\frac{e_{g 1}^{U 2}}{\left(1+e_{g 1}^{U}\right)^{2}} \frac{a}{8}\left[\left(1+\frac{e_{g 1}^{U}}{\sqrt{2}}\left(\sqrt{\frac{2 \sqrt{2}}{e_{g 1}^{U}}+1}-1\right)\right)^{2}\left(1+e_{g 1}^{U}\right)^{2}-16\left(e_{g 1}^{U}+\frac{1}{a}+\frac{\Delta^{2}}{2 a^{2}}\right)\right] .
$$

Let $\Delta \pi(a, \Delta)=\frac{\Delta \Pi(a, \Delta)}{q}$. Note that independently from $\Delta \in[0,5], \lim _{a \rightarrow+\infty} \Delta \pi>0$ as $\lim _{a \rightarrow+\infty}\left(e_{g 1}^{U}\right)^{2} \frac{a}{8}=\frac{1}{2}$. By continuity it is still true for lower values of $a$. When $a$ is very large so that success is difficult, the firm's profit is always higher with up-or-out than with standard promotion practices. 
We now prove that for low values of $\Delta$ it exists $a^{l}(\Delta)>37.5$ such that $\Delta \pi(a, \Delta) \geq 0$ if and only if $a \geq a^{l}(\Delta)$. Let $f(a, \Delta)=\left(1+\frac{e_{g 1}^{U}}{\sqrt{2}}\left(\sqrt{\frac{2 \sqrt{2}}{e_{g 1}^{U}}+1}-1\right)\right)^{2}\left(1+e_{g 1}^{U}\right)^{2}$ and $d(a, \Delta)=$ $4 \sqrt{2} \frac{\Delta}{a}\left(1+e_{g 1}^{U}\right) \frac{2 \sqrt{2}-1-e_{g 1}^{U}}{e_{g 1}^{U}}$. From (45) $\Delta \pi(a, \Delta)>0$ with $a \geq 37.5$ and $\Delta \in(0,5]$ is equivalent to

$$
f\left(e_{g 1}^{U}(a, \Delta)\right)+d(a, \Delta)>16\left(e_{g 1}^{U}(a, \Delta)+\frac{1}{a}+\frac{\Delta^{2}}{2 a^{2}}\right)
$$

This part of the proof proceeds in two steps. In step 1 we consider the case $\Delta=0$ and show that there exists a unique $a^{l}(0)>37.5$. In step 2: we consider the case $\Delta>0$. We show that there is a critical value $\Delta^{l}$ such that for all $\Delta<\Delta^{l}$ it exists $a^{l}(\Delta)>37.5$ so that $\Delta \pi \geq 0$ if and only if $a \geq a^{l}(\Delta)$. To conclude we show that for all $\Delta>\Delta^{l}$ we have $\Delta \pi>0$ for all $a \geq 37.5$.

Step 1: Consider the case $\Delta=0$ so that $e_{g 1}^{U}=\frac{2}{\sqrt{a}}$ and $d(a, \Delta)=0$. From (46) $\Delta \Pi>0$ is equivalent to $f\left(\frac{2}{\sqrt{a}}\right)>16\left(\frac{2}{\sqrt{a}}+\frac{1}{a}\right)$. The functions $f\left(\frac{2}{\sqrt{a}}\right)$ and $16\left(\frac{2}{\sqrt{a}}+\frac{1}{a}\right)$ can cross only once, twice or none because they are both decreasing and convex in $a$. Since $f\left(\frac{2}{\sqrt{37.5}}\right)<16\left(\frac{2}{\sqrt{37.5}}+\frac{1}{37.5}\right)$, while $\lim _{a \rightarrow+\infty} f\left(\frac{2}{\sqrt{a}}\right)=1>\lim _{a \rightarrow+\infty} 16\left(\frac{2}{\sqrt{a}}+\frac{1}{a}\right)=0$ they cross once. There is a unique $a^{l}>37.5$ so that $\Delta \pi \geq 0$ if and only if $a \geq a^{l}$. We have $a^{l} \sim 182.95$ for $\Delta=0$.

Step 2: We now turn to the case $\Delta>0$. One can check after tedious computations that for all $\Delta \in(0,5], f\left(e_{g 1}^{U}(a, \Delta)\right)$ and $d(a, \Delta)$ are decreasing and convex in $a \geq 37.5$. Therefore, $f\left(e_{g 1}^{U}(a, \Delta)\right)+d(a, \Delta)$ is decreasing and convex in $a$. We can thus apply the same reasoning as before. Since $\lim _{a \rightarrow+\infty} \Delta \pi(a, \Delta)>0 \forall \Delta \in[0,5]$, we just need to show that it exists a $\Delta^{l}>0$ so that $\Delta \pi(37.5, \Delta)<0 \forall \Delta<\Delta^{l}$. The function $\Delta \pi(37.5, \Delta)$ is continuous in $\Delta$. Moreover $\Delta \pi(37.5,0)<0$ while $\Delta \pi(37.5,5)>0$ so that there is at least one $\Delta^{l} \in(0,5]$ so that $\Delta \pi(37.5, \Delta)=0$. The following graph of $\Delta \pi(37.5, \Delta)$ shows that $\Delta^{l}$ is unique with $\Delta^{l} \sim 2.07$.

Figure 1 about here.

Finally, note that by construction $\Delta \pi(37.5, \Delta)<0$ for all $\Delta<\Delta^{l}$. Hence, for all $\Delta<\Delta^{l}$ it exists $a^{l}(\Delta)>37.5$ so that $\Delta \pi \geq 0$ if and only if $a \geq a^{l}(\Delta)$. The following graph shows that for $\Delta>\Delta^{l}$ we have $\Delta \pi>0$ for all $a \geq 37.5$.

Figure 2 about here. 
Figure 1

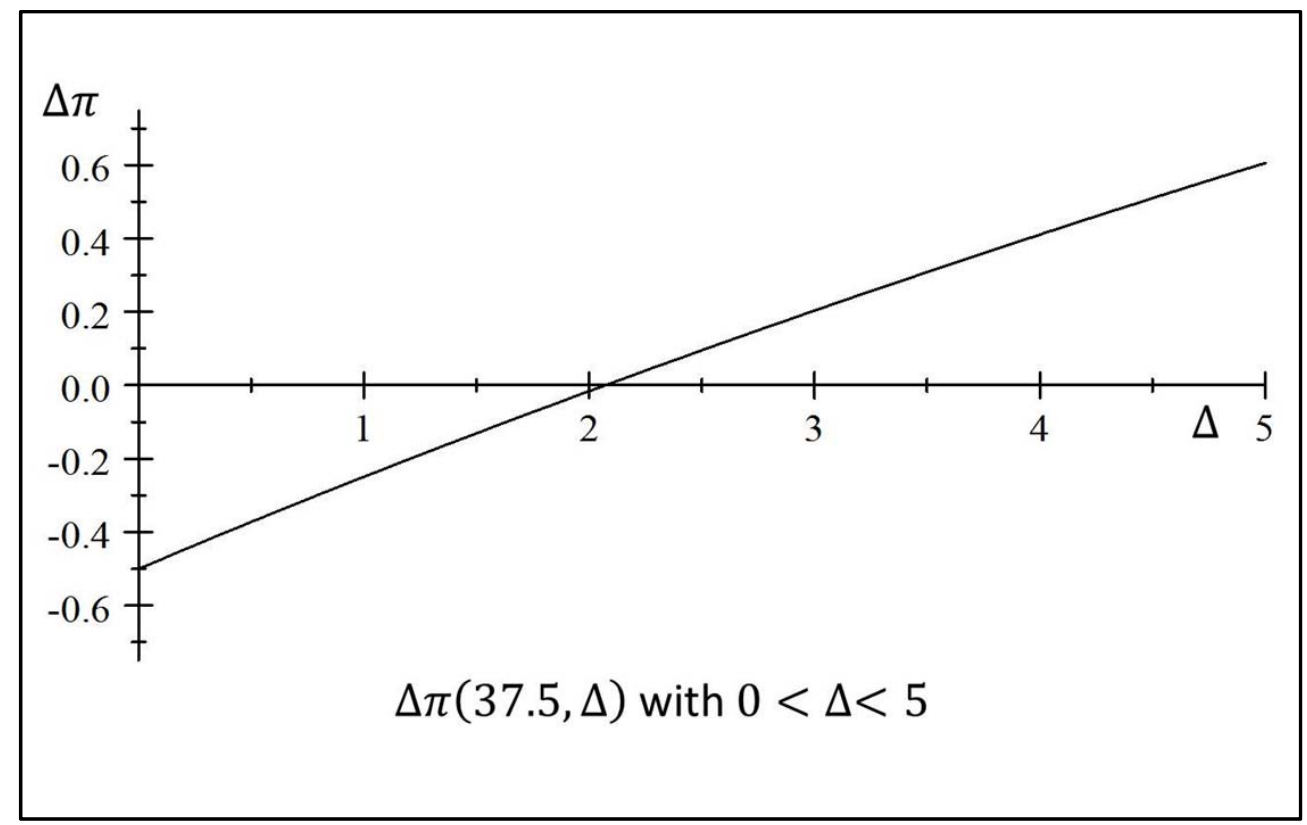

Figure 2

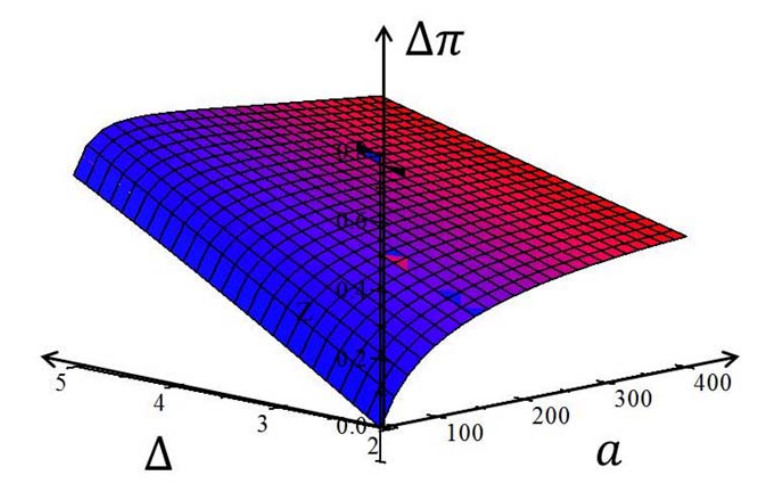

$\Delta \pi(a, \Delta)$ with $a>37.5$ and $\Delta^{l}<\Delta<5$ 